\title{
Déploiement d'un réseau de radars pour anticiper les risques hydrométéorologiques
}

\author{
Samuel Westrelin(1), Patrice Mériaux ${ }^{(2)}$, Serge Dalle(3), Béatrice Fradon ${ }^{(4)}$, Gérard Jamet(3)
}

(1) Météo-France, Direction interrégionale Sud-Est, Irstea Aix-en-Provence, Unité de recherche hydrobiologie, 3275 route de Cézanne, CS 40061, 13182 Aix-en-Provence Cedex 5

(2) Irstea Aix-en-Provence, Unité de recherche Ouvrages hydrauliques et hydrologie, 3275 route de Cézanne, CS 40061, 13182 Aix-en-Provence Cedex 5

(3) Météo-France, Direction des systèmes d'observations, Centre de météorologie radar, 7 rue Teisserenc-de-Bort, BP 202, 78195 Trappes

(4) Météo-France, Direction des systèmes d'observations, Centre de météorologie radar, 42 avenue Gaspard Coriolis, 31057 Toulouse Cedex

\section{Résumé}

Cet article décrit les grandes lignes du projet Rhytmme ${ }^{1}$ qui s'étale sur la période 2008-2014 et contribue à la gestion des risques naturels liés aux précipitations en région Provence-AlpesCôte d'Azur (inondations, crues torrentielles, glissements de terrain, chutes de bloc, avalanches, feux de forêt). Ses objectifs sont, d'une part, de compléter la couverture du réseau national de radars de Météo-France sur les Alpes du Sud avec des radars de nouvelle technologie et, d'autre part, de développer une plateforme multirisques, accessible en ligne, pérenne, avertissant en temps réel les autorités gestionnaires des risques naturels quant à l'occurrence probable d'un ou plusieurs aléas.

\section{Abstract \\ Installation of a radar network to anticipate hydrometeorological risks}

This paper describes the broad lines of the Rhytmme (Hydrometeorological hazards in Mediterrranean mountainous areas) project which covers the 2008-2014 period and contributes to the management of natural hazards related to precipitation in the Provence-Alps-Côte d'Azur region (flash floods, debris flows, landslides, rockfalls, snow avalanches, forest fires). It aims at, on one hand, improving the national radar network coverage over the Southern Alps with radars using new technologies and, on the other hand, developing a perennial multi-hazard web platform warning authorities in charge of natural hazard management when an event might occur.

\section{Introduction}

Les quelque 1000 communes de la région Provence-Alpes-Côte d'Azur sont exposées à au moins l'un des cinq aléas suivants : crues éclairs, laves torrentielles ${ }^{2}$, mouvements de terrain, avalanches et feux de forêts. Dans cette région densément peuplée sur sa zone littorale et dans ses principales vallées, ces aléas engendrent des risques importants pour les populations et les infrastructures. Les trois départements de montagne au relief très escarpé, culminant à plus de $3500 \mathrm{~m}$, peuvent être l'objet de précipitations intenses et contrastées, exposant une centaine de communes à l'ensemble de ces cinq aléas. L'occurrence et l'intensité de ces aléas dépendent plus ou moins directement de la quantité et de la nature des précipitations. Or, sur

\footnotetext{
${ }^{1}$ Risques hydrométéorologiques en territoires de montagnes et méditerranéens.

2 Écoulements d'un mélange de boues et d'éléments rocheux dans des lits à forte pente, supérieure à 7 \%, caractéristiques des torrents de montagne.
} 
l'arc alpin français, la visibilité des radars du réseau national Aramis de Météo-France est considérablement réduite par les effets de masque du relief, d'échos fixes et d'altitude du faisceau. En conséquence, l'estimation des cumuls de pluie y est de qualité médiocre.

Dans ce contexte, le projet Rhytmme (Mériaux et al., 2011 ; Westrelin et al., 2012), piloté conjointement par Météo-France et l'Irstea ${ }^{3}$ sur la période 2008-2014, s'attèle à :

- $\quad$ compléter le réseau de radars existant par le déploiement dans les Alpes du Sud de radars de nouvelle technologie (radars Doppler polarimétriques en bande $\mathbf{X}$ ) afin d'estimer en temps réel les quantités de pluie à une résolution spatiotemporelle élevée $\left(1 \mathrm{~km}^{2}\right.$, pas de temps de 5 min) ;

- développer, en aval de ces mesures radar, une plateforme de services d'aide à la gestion en temps réel des risques naturels hydrométéorologiques, notamment en montagne.

Cet article présente tout d'abord la stratégie adoptée pour améliorer la couverture radar dans les Alpes du Sud, en se focalisant sur les systèmes radar choisis ainsi que sur l'ingénierie déployée pour en traiter les mesures. Il expose ensuite les applications aval en temps réel qui découlent du réseau de radars installé et leur expérimentation par les gestionnaires des risques naturels.

\section{La démarche de déploiement du réseau de radars Bases scientifiques}

Dès la fin des années 1960, les bénéfices potentiels du radar sont perçus en hydrométéorologie et des expérimentations sont menées avec un radar bande $\mathrm{X}$ dans un service d'annonce des crues pour quantifier et spatialiser les pluies et ainsi mieux apprécier les probabilités de crue (Dupouyet, 1983).

La faible longueur d'onde des radars bande X $(3 \mathrm{~cm})$ permet de réduire significativement la taille d'antenne par rapport à celle des radars bande $\mathbf{S}$ ou $\mathbf{C}$ (d'un facteur 3 à 4 en diamètre) et en fait un outil intéressant pour compléter les réseaux en place composés de ces radars. Cette stratégie a d'abord été explorée en zone urbaine soumise à des aléas hydrologiques importants. Le relief alentour était alors utilisé pour quantifier l'atténuation et corriger les mesures (Delrieu et Creutin, 1991 ; Delrieu et al., 1997 ; Delrieu et al., 1999a). L'utilisation du radar en zone à relief a aussi entraîné des recherches d'une part sur l'effet de la structure verticale de l'atmosphère sur la mesure (Andrieu et Creutin, 1995 ; Andrieu et al., 1995) et d'autre part sur la visibilité hydrologique qui quantifie la qualité de la mesure par un calcul numérique combinant géométrie de la mesure, structure verticale de l'atmosphère et topographie (Pellarin et al., $2000 ; 2002$ ).

Mais l'atténuation dont souffrent particulièrement les mesures en bande X (Delrieu et al., 1999b, 2000) a longtemps été un obstacle à une utilisation plus répandue de ce type de radar.

Pourtant, dès 1983, Sauvageot écrit à propos des estimations de précipitations par radar : «[...] Parmi les voies d'études nouvelles, les plus prometteuses semblent être la prise en compte de l'état de polarisation des signaux rétrodiffusés qui apporte une information supplémentaire sur la taille des hydrométéores [...] ». Dans les années 2000, les recherches sur la polarimétrie débouchent sur des algorithmes permettant de s'affranchir en bonne partie de l'atténuation (par exemple, Testud et al., 2000 ; Le Bouar et al., 2001 ; Bringi et Chandrasekar, 2001) et devenus mûrs pour servir des applications opérationnelles (Testud, 2002).

Depuis, l'utilisation des radars bande X connaît un essor dans le monde pour compléter les réseaux existants ou soutenir des applications locales (revue dans Cremonini et al., 2012), comme par exemple en Allemagne (Feiertag et al., 2012), au Japon (Maesaka et al., 2011), aux États-Unis (McLaughlin et al., 2009 ; Palumbo et al., 2012).

\footnotetext{
${ }^{3}$ Institut national de recherche en sciences et technologies pour l'environnement et l'agriculture (ex. Cemagref)
} 
En France, un prototype industriel (Hydrix $®)$ de radar Doppler polarimétrique en bande X a fait l'objet de tests dans une zone soumise à de fortes précipitations (Diss et al., 2006 ; 2009). L'algorithme polarimétrique utilisé pour l'estimation des précipitations (Testud et al., 2000) a permis de corriger en bonne partie le signal atténué dans un rayon de $60 \mathrm{~km}$. Des cas d'atténuation ont été observés même dans un rayon de $30 \mathrm{~km}$ du radar lors de précipitations intenses et des cas d'extinction totale ne peuvent pas être écartés (Tabary et al., 2008). En juin 2007, le radar Hydrix®, propriété du CNRS et exploité par la société Novimet, a été installé au mont Vial (alt. $1550 \mathrm{~m}$ ), à $20 \mathrm{~km}$ au nord de Nice dans les Alpes-Maritimes pour servir des applications hydrométéorologiques.

\section{La stratégie adoptée}

Le réseau de radars Aramis actuel (Tabary et al., 2013a), constitué de radars bande C et S, dont le plus élevé culmine à $1116 \mathrm{~m}$, a une visibilité médiocre sur les Alpes du Sud, en partie à cause du masque que constituent les versants montagneux. L'étude menée à partir du radar Hydrix® constitue une étude de faisabilité de Rhytmme et ses données nourrissent le projet depuis janvier 2010 ; ce radar complète la mosaïque Aramis sur l'extrême sud-est. Outre la diminution des coûts qu'elle engendre sur le radar lui-même et sur ses infrastructures, la compacité des radars en bande $X$, moins sensibles aux échos de sol que les autres radars (Delrieu et Creutin, 1991), les rend bien adaptés à une installation en milieu montagneux. Sur ces bases, la stratégie adoptée dans Rhytmme a été de compléter la couverture radar des régions montagneuses vulnérables de la Région Provence-Alpes-Côte d'Azur par des radars Doppler polarimétriques en bande $\mathrm{X}$ mais en favorisant une installation en réseau avec un large chevauchement des zones de visibilité entre deux radars voisins, distants au maximum d'environ $60 \mathrm{~km}$, pour prévenir au mieux des phénomènes de forte atténuation voire d'extinction et aussi combler les nombreux masques liés aux versants montagneux.

L'exploitation optimale des mesures de ces radars de nouvelle technologie a nécessité le développement de nouveaux algorithmes de traitement du signal (Tabary et al., 2013b), précurseurs de la future chaîne opérationnelle radar de Météo-France d'ici 2014.

On peut souligner que cette démarche française de compléter un réseau de radars bande $\mathrm{C}$ et $\mathrm{S}$ par des radars bande $\mathrm{X}$ est originale. En effet, des pays voisins également montagneux font le choix de la bande C, comme par exemple la Suisse (Germann et al., 2012) et l'Autriche (Kaltenboeck, 2012), alors que la bande C souffre également d'atténuation même si elle est moins prononcée (Delrieu et al., 1999b) et nécessite des installations beaucoup plus volumineuses.

\section{La mise en place du réseau de radars, ses caractéristiques et son exploitation Les contraintes d'installation en milieu montagneux}

Les critères de sélection des sites potentiels d'accueil de radar étaient les suivants :

- offrir une bonne visibilité sur les zones à enjeux définies: le calcul de la visibilité hydrologique (Pellarin et al., 2000 ; 2002) du site radar sélectionné permet de se faire une première idée de la qualité potentielle du site ; elle est obligatoirement complétée d'une visite sur place qui permet de se rendre compte de la présence d'obstacles anthropiques (arbres, pylônes...) ;

- pouvoir être relié relativement facilement aux réseaux (électricité, télécommunications) ;

- pouvoir accéder au site toute l'année, notamment pour la construction des infrastructures et la maintenance ultérieure des radars ;

- prévenir toute difficulté administrative d'installation (zones à enjeux écologiques, économiques, identification du ou des propriétaires du terrain...) ;

- éviter les altitudes trop élevées pour limiter les contraintes de maintenance.

La réalité du terrain montagneux s'est très rapidement imposée et il s'est en fait agi de trouver le meilleur compromis entre ces différentes contraintes. 
Author-produced version of the article published in La Meteorologie, 2013, 83, 8, pp. 69-79

Original publication available at http://documents.irevues.inist.fr/handle/2042/52056

doi : $10.4267 / 2042 / 52056$

Trois radars Doppler polarimétriques en bande X ont été installés dans le cadre de Rhytmme (les altitudes données ci-dessous sont celles du faisceau du radar) : un premier radar au sommet de la montagne Maurel $(1779 \mathrm{~m})$ sur la commune de La Mure-Argens (Alpes-deHaute-Provence) en octobre 2010 (figure 1) ; un deuxième au mont Colombis (1742 m) sur la commune de Théus (Hautes-Alpes) en octobre 2012 (figure 2) ; un troisième au sommet de Vars Mayt (2581 m) sur la commune de Vars (Hautes-Alpes) en juillet 2013 (figure 3).

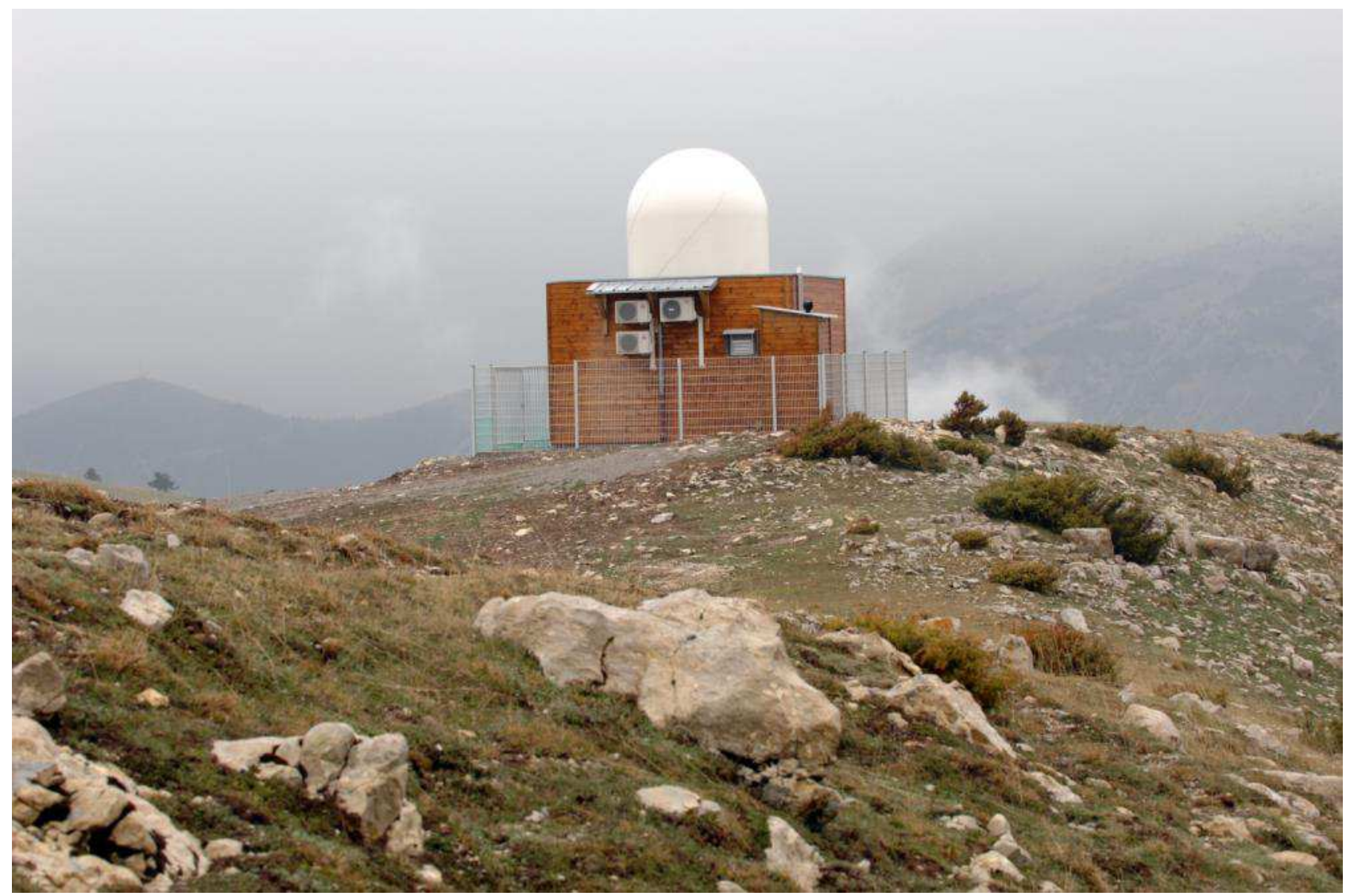

Figure 1. Installation radar au sommet de la montagne Maurel à $1779 \mathrm{~m}$ (altitude du faisceau radar), sur la commune de La Mure-Argens dans les Alpes-de-Haute-Provence. (CMétéo-France, Pascal Taburet. 


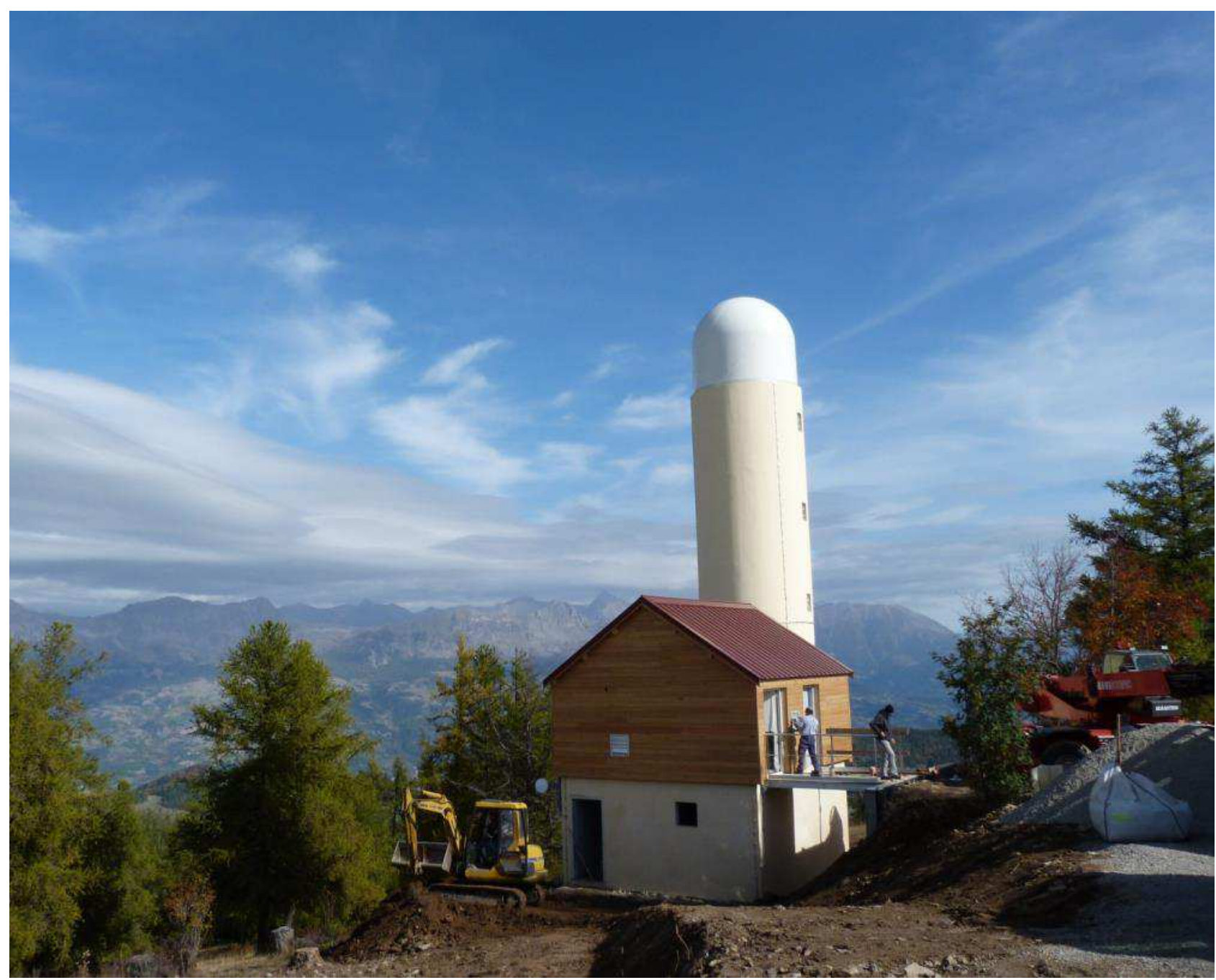

Figure 2. Installation radar au sommet du mont Colombis à $1742 \mathrm{~m}$ (altitude du faisceau radar), sur la commune de Théus dans les Hautes-Alpes. @Météo-France, Yves Clémenceau. 


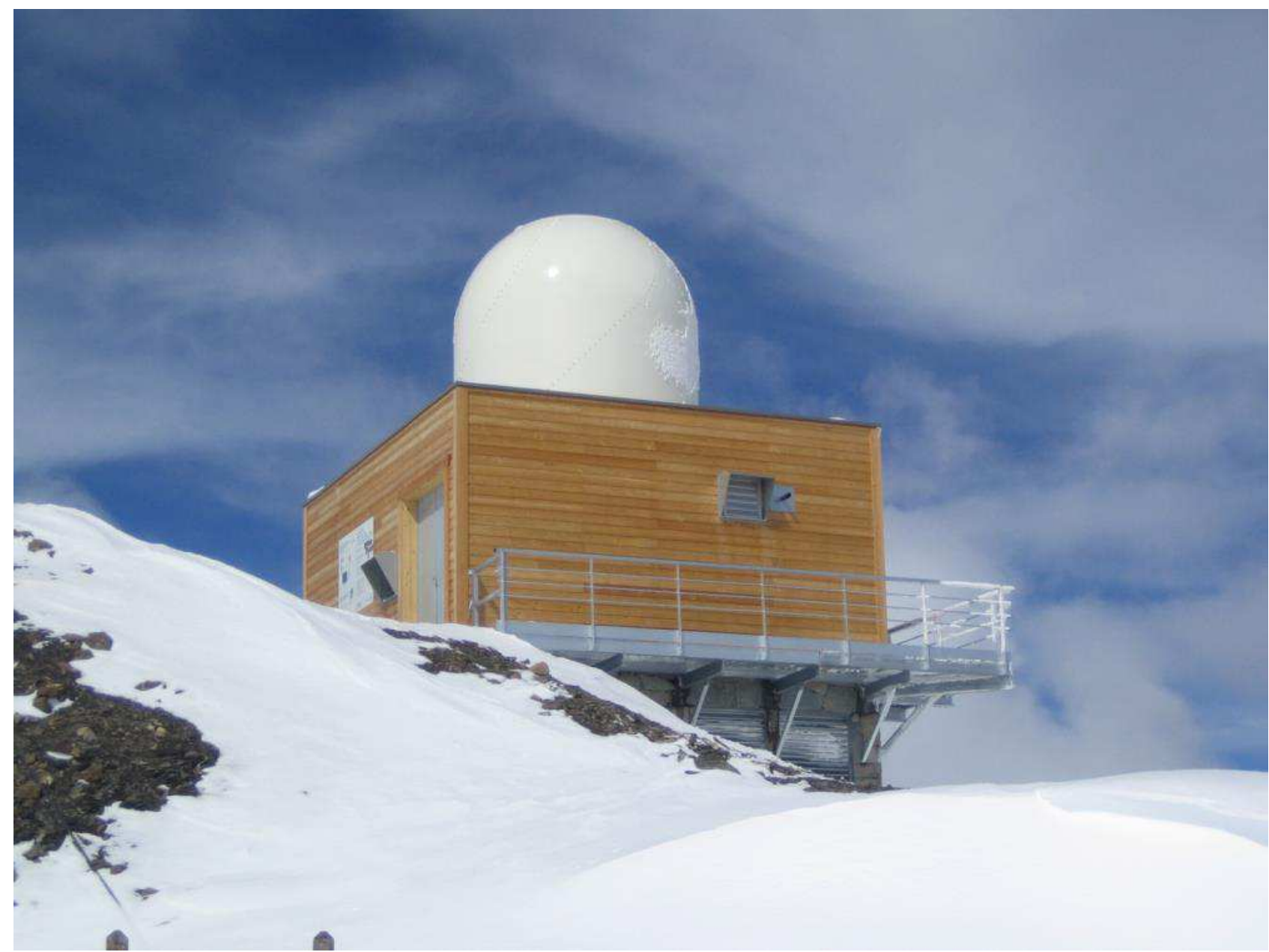

Figure 3. Installation radar au sommet de Vars Mayt à $2581 \mathrm{~m}$ (altitude du faisceau radar), sur la commune de Vars dans les Hautes-Alpes. (C) Météo-France, Yves Clémenceau.

Pour chacun de ces sites, une étude d'impact environnemental a été menée pour vérifier que la construction ne menaçait pas d'espèces floristiques ou faunistiques sensibles. Les entreprises de travaux ont suivi une démarche de respect environnemental ou une charte de chantier vert. Enfin, la bonne intégration paysagère des infrastructures a fait l'objet d'une attention particulière, celles-ci étant par exemple toutes recouvertes d'un bardage bois. Les bâtiments sont relativement réduits et d'une emprise au sol d'environ $30 \mathrm{~m}^{2}$ contenant un local technique et un local groupe électrogène avec le radar installé sur le toit terrasse, excepté au mont Colombis, où les arbres et forêts environnants nous ont contraints à installer le radar au sommet d'une tour de $19 \mathrm{~m}$ de haut.

La cartographie globale du réseau de radars de Météo-France, excluant le radar du mont Vial, est représentée dans Tabary et al. (2013a). La figure 4a montre une modélisation de la couverture hydrologique radar de la région Provence-Alpes-Côte d'Azur obtenue en combinant les radars du réseau Aramis, ceux du mont Vial et de la montagne Maurel. La quasitotalité du département des Alpes-Maritimes, ainsi qu'une grande moitié sud du département des Alpes-de-Haute-Provence y apparaissent relativement bien couvertes. Cependant, les versants montagneux élevés masquent de nombreux secteurs au nord du radar de la montagne Maurel et illustrent les contraintes du terrain montagneux ; tel est le cas du secteur nord-nord-ouest masqué par la montagne du Cheval Blanc, qui culmine à plus de $2300 \mathrm{~m}$ en pays dignois. Dans un tel contexte, l'installation en réseau rapproché prend alors tout son sens : le radar du mont Colombis, installé à environ $60 \mathrm{~km}$ au nord-nord-ouest de la montagne Maurel, vient pallier la mauvaise visibilité du réseau précédent dans de nombreux secteurs 
Author-produced version of the article published in La Meteorologie, 2013, 83, 8, pp. 69-79

Original publication available at http://documents.irevues.inist.fr/handle/2042/52056

doi : $10.4267 / 2042 / 52056$

(figure 4b). La plupart de ses propres secteurs masqués sont eux-mêmes visibles du radar de Vars. 
Author-produced version of the article published in La Meteorologie, 2013, 83, 8, pp. 69-79

Original publication available at http://documents.irevues.inist.fr/handle/2042/52056

doi : $10.4267 / 2042 / 52056$

a)

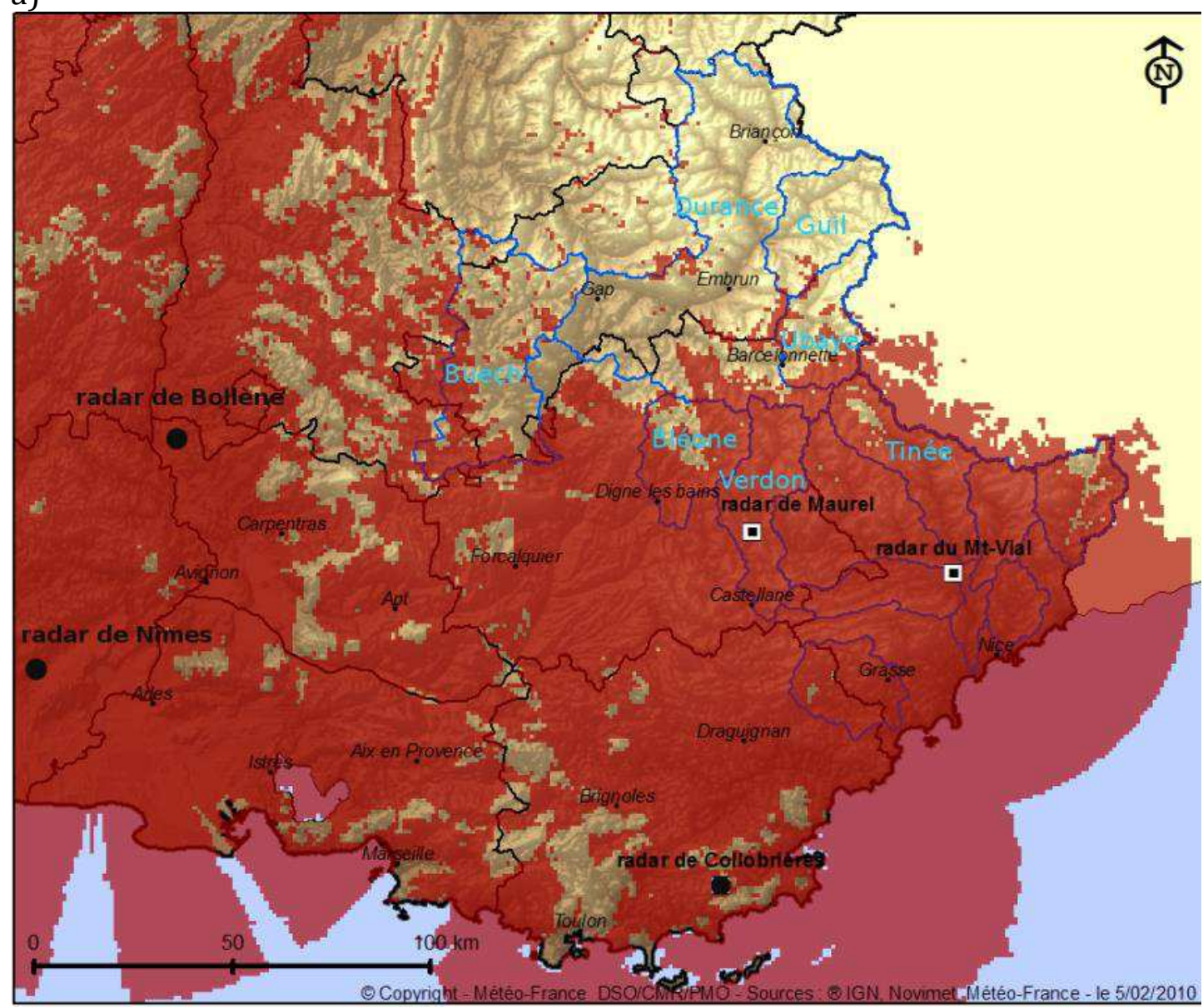

b)

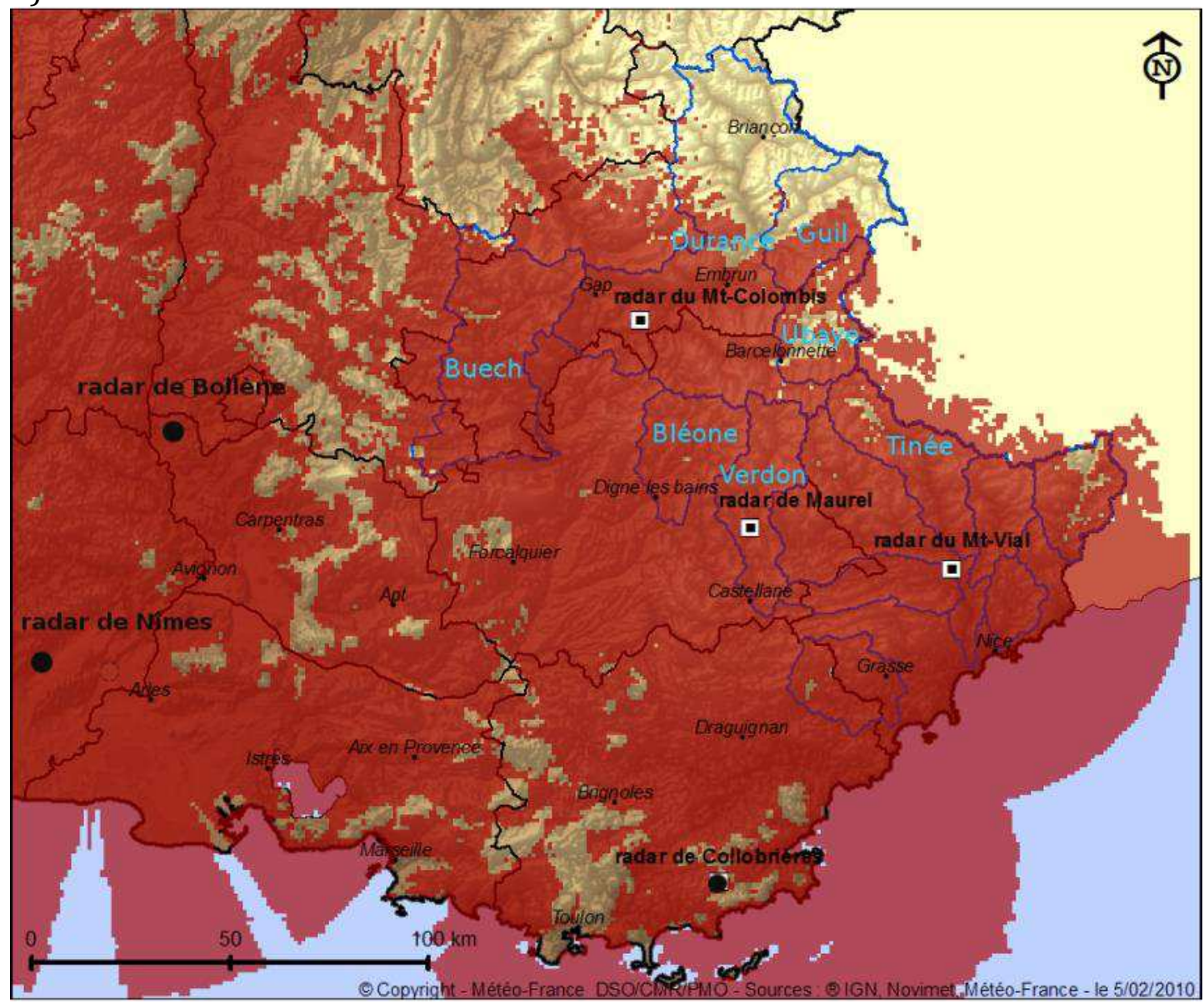


Figure 4. a. Visibilité hydrologique du réseau de radars Aramis complété du radar Hydrix® et du radar de la montagne Maurel, dans le sud-est de la France. Les codes de qualité Aramis supérieurs ou égaux à 84 (sur une échelle allant de 0 à 100) sont représentés en rouge ; ils correspondent aux zones où l'estimation des cumuls de pluie par le réseau radar est la meilleure. Les contours des principaux bassins versants d'intérêt sont tracés en bleu ; les pastilles noires symbolisent l'emplacement des radars bande $\mathrm{S}$ du réseau Aramis et les carrés noirs à contour blanc les radars bande X. b. Identique, avec en plus le radar polarimétrique en bande $\mathrm{X}$ installé au Mont Colombis.

L'accessibilité des installations est variable selon les sites et les saisons: la première (montagne Maurel) est au sommet d'une piste de $10 \mathrm{~km}$ très caillouteuse, mais relativement carrossable de mai à octobre, la deuxième (mont Colombis) au sommet d'une route partiellement déneigée l'hiver et la troisième (Vars) au sommet des remontées mécaniques d'une station de ski. Un volant d'heures d'hélicoptère de la sécurité civile est mobilisable pour accéder à ces sites en conditions hivernales et en cas de besoin urgent de maintenance curative. En cas de panne électrique, un groupe électrogène prend le relais; il est dimensionné pour assurer en théorie quatre jours d'autonomie de l'ensemble de l'installation à pleine puissance, soit une semaine environ en pratique, puisque toutes les installations électriques ne fonctionnent pas simultanément.

\section{Les caractéristiques techniques des radars installés}

Le système radar du projet Rhytmme est fourni par la société Selex. Il s'agit d'un radar Doppler à double polarisation en bande $\mathrm{X}$ de dernière génération, fonctionnant dans la bande 9450 à $9500 \mathrm{MHz}$. Ce radar est la version «fixe» du modèle mobile Météor $50 \mathrm{DX}$, radar initialement destiné à la recherche (il a, notamment, participé à la campagne Framea (Diss et al., 2006), dans le Piémont italien). Sa principale caractéristique, qui le distingue des radars "classiques", est l'intégration de l'ensemble émission/réception et motorisation (commandes d'antenne) sur l'antenne. Cette conception permet de réduire notablement l'encombrement de l'ensemble (notamment du fait de l'absence d'armoire électronique), ce qui est un avantage pour une installation en région montagneuse où l'espace disponible est compté. Comme le montre la figure 5, l'émetteur et le récepteur analogique, d'une part, et la commande de motorisation, le récepteur numérique et le processeur de contrôle radar, d'autre part, sont situés sur la partie tournante de la tourelle, à l'emplacement habituel des contre-poids. 


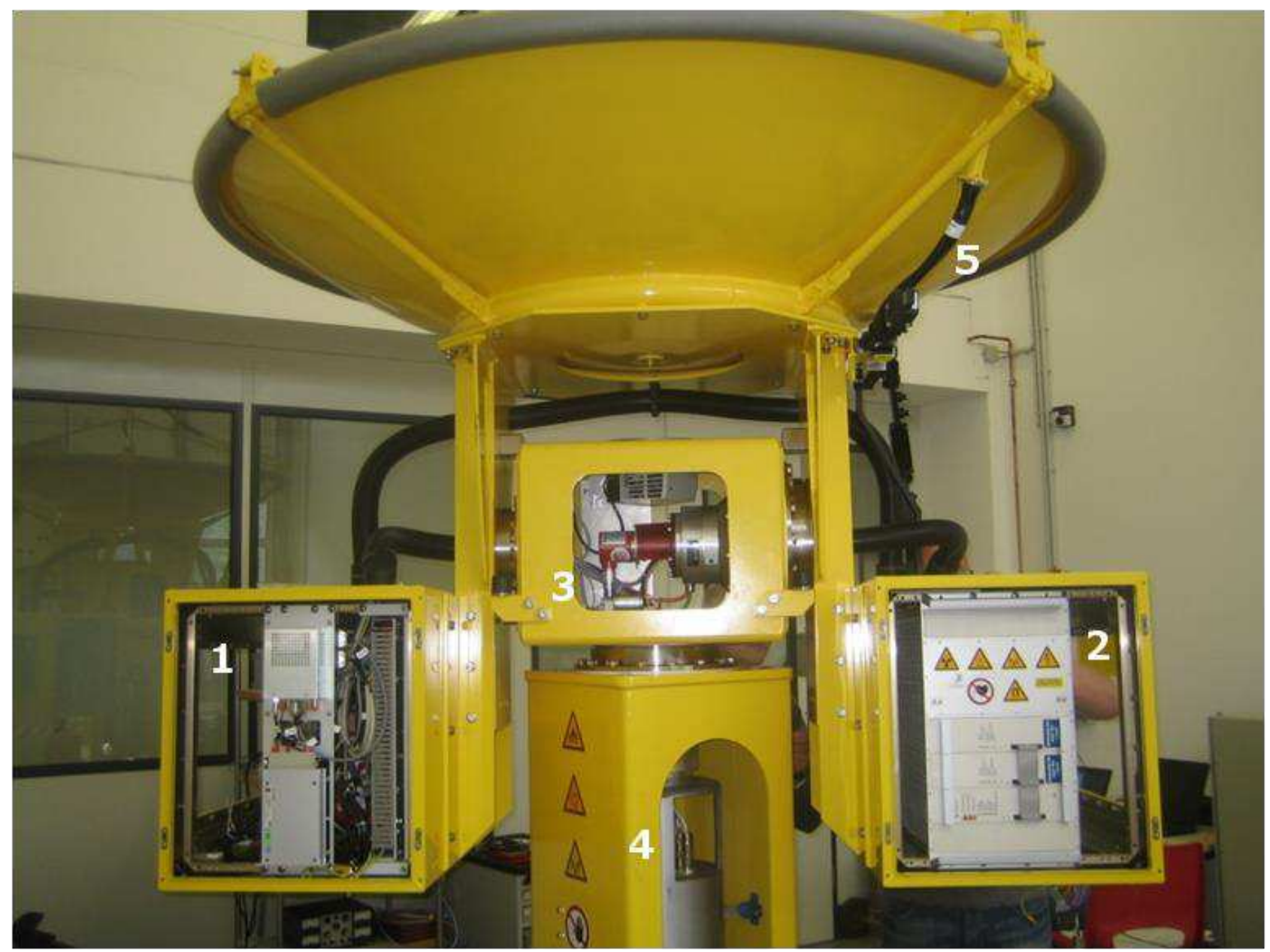

Figure 5. Radar doppler polarimétrique en bande X (modèle Selex Météor 50 DX) installé dans le cadre du projet Rhytmme. Le diamètre de l'antenne est de $1,80 \mathrm{~m}$. 1 : processeur de contrôle radar ; 2 : émetteur-récepteur ; 3 : boîtier de contrôle du site ; 4 : boîtier azimutal ; 5 : guide d'onde et antenne. La rotation dans les plans horizontal (4) et vertical (3) permet une exploration volumique de l'atmosphère. L'émetteur (2) génère une impulsion électromagnétique de forte puissance, de l'ordre de $70 \mathrm{~kW}$, qui est transmise à l'antenne via le trajet du guide d'onde (5). Cette impulsion électromagnétique est réfléchie et retournée vers le radar par les cibles que constituent les hydrométéores présents dans l'atmosphère. L'intensité du signal retourné au radar est fonction de la densité et de la surface de réflectivité ces cibles. L'antenne (5) amplifie le signal reçu avant de le transmettre au récepteur (1 et 2), toujours via le trajet du guide d'onde, qui le convertit en signal électrique analysable par le calculateur Castor. Photo (C Serge Dalle, Météo-France.

Un autre avantage de cette solution réside dans le fait que les joints tournants (le joint tournant est une pièce métallique entraînée par les mouvements de l'antenne par laquelle transite l'onde radio) en site (angle d'inclinaison de l'antenne par rapport à la verticale) et azimut (angle d'inclinaison de l'antenne par rapport au Nord géographique) sont supprimés du trajet du guide d'onde.

L'ensemble étant particulièrement compact et localisé dans un espace sous radôme dont la température ne peut être qu'imparfaitement régulée (l'altitude des différents sites s'étend de $1700 \mathrm{~m}$ à $2600 \mathrm{~m}$ ), l'industriel a dû mettre en place des solutions classiques (ventilation, réchauffage automatique), mais aussi innovantes (refroidissement par effet Peltier ${ }^{4}$ ) pour limiter l'influence de la température et de l'humidité sur les performances de l'électronique.

\footnotetext{
${ }^{4}$ Phénomène physique de déplacement de chaleur en présence d'un courant électrique. L'effet se produit dans des matériaux conducteurs de natures différentes liés par des jonctions (contacts). Une des jonctions se refroidit alors légèrement, pendant que l'autre se réchauffe.
} 
Author-produced version of the article published in La Meteorologie, 2013, 83, 8, pp. 69-79

Original publication available at http://documents.irevues.inist.fr/handle/2042/52056

doi : $10.4267 / 2042 / 52056$

Les caractéristiques techniques affichées par le constructeur couvrent une gamme large de températures $\left(-25^{\circ} \mathrm{C}\right.$ à $\left.+55^{\circ} \mathrm{C}\right)$ et d'humidités relatives $(0-100 \%)$.

La réception reprend le récepteur numérique GDRX® (qui fournit le signal de synchronisation à l'ensemble du radar et effectue l'acquisition et l'échantillonnage du signal en fréquence intermédiaire issu du récepteur analogique) tandis que l'émission reprend le concept de modulateur " état solide » (le signal de déclenchement de l'émission n'est plus fourni par un tube à hydrogène, comme le thyratron, mais par une électronique de puissance à semiconducteur), deux solutions développées par Selex et largement utilisées sur les modèles Météor 510 C, Météor $600 \mathrm{C}$ et Météor $600 \mathrm{~S}$ opérant dans le réseau Aramis. Le radar seul requiert une puissance électrique inférieure à $5 \mathrm{~kW}$ pour son fonctionnement en continu.

La tenue au vent et la protection de l'antenne contre les dépôts de neige ou de glace, contraintes préjudiciables au bon fonctionnement de la mécanique, sont assurées par un radôme (diamètre 3,50 $\mathrm{m}$ ) fourni avec le radar et assemblé sur le site. Ce radôme est conçu pour résister à des vents maximaux de $60 \mathrm{~m} / \mathrm{s}$ (soit $216 \mathrm{~km} / \mathrm{h}$ ). Un des inconvénients du radôme est que le film d'eau qui le recouvre en cas de pluie atténue le signal mesuré par le radar. Cependant, cette atténuation peut être relativement bien quantifiée (Frasier et al., 2013), et une correction peut donc être envisagée dans les algorithmes temps réel, et certains algorithmes polarimétriques, notamment ceux qui se basent sur la phase du signal radar (Tabary et al., 2013b), y sont insensibles.

Le calculateur Castor3, assemblé et fourni par Selex, est une version « allégée » du calculateur Castor2 conçu par l'équipe technique de Météo-France. Il reprend l'essentiel des fonctions de son aîné (traitement du signal, élaboration de produits de réflectivités, de vitesses Doppler, de lames d'eau, commandes des modes d'exploration de l'atmosphère, acquisition des paramètres de fonctionnement du radar...), mais le déport vers le radar de certaines d'entre elles (étalonnage du récepteur, mesure de la puissance émise) permet de réduire d'un facteur deux à trois les dimensions du calculateur hébergé dans le local technique, une des contraintes du projet Rhytmme étant de limiter au mieux l'emprise au sol des équipements techniques.

Les données issues du récepteur numérique sont transmises au calculateur Castor3 via le réseau filaire local par lequel transitent également les paramètres de fonctionnement et les éventuelles alarmes du radar.

Intégrée à ce sous-réseau informatique, une centrale de surveillance utilisant le protocole Internet renseigne le calculateur sur l'état de l'environnement (température dans le local technique, sous le radôme, présence du secteur, etc.). Ces informations sont ainsi accessibles à distance, notamment par les services de maintenance, ce qui permet à la fois de limiter les visites de contrôle et de prévenir d'éventuels dysfonctionnements ou pannes majeures avec une meilleure réactivité.

Tableau 1. Récapitulatif des caractéristiques techniques du radar installé dans Rhytmme et de son radôme.

\begin{tabular}{|c|c|}
\hline Poids du radar & $800 \mathrm{~kg}$ \\
\hline Diamètre de l'antenne & $1,80 \mathrm{~m}$ \\
\hline Vitesse max en site et azimut & $36^{\circ} / \mathrm{s}$ \\
\hline Angle d'ouverture & $<1,35^{\circ}$ \\
\hline Gain de l'antenne & $>42 \mathrm{~dB}$ \\
\hline Poids du radôme & $250 \mathrm{~kg}$ \\
\hline Diamètre du radôme à l'équateur & $3,50 \mathrm{~m}$ \\
\hline
\end{tabular}


Author-produced version of the article published in La Meteorologie, 2013, 83, 8, pp. 69-79

Original publication available at http://documents.irevues.inist.fr/handle/2042/52056

doi : $10.4267 / 2042 / 52056$

\begin{tabular}{|c|c|}
\hline Facteur de bruit du récepteur & $<2,5 \mathrm{~dB}$ \\
\hline Dynamique de réception & $90 \mathrm{~dB}$ \\
\hline Puissance crête de l'émetteur & $75 \mathrm{~kW}$ nominal \\
\hline Fréquence de répétition maximale & $2 \mathrm{kHz}$ \\
\hline Largeur d'impulsion & $0,5 \mu \mathrm{s}$ min à $2 \mu \mathrm{s} \max$ \\
\hline
\end{tabular}

Ces radars effectuent des mesures en temps réel selon un cycle bien défini (nombre de tours par minute à chaque site prospecté et pour chaque fréquence de répétition des impulsions radar), dit "mode d' exploitation», dicté par les applications en aval du radar. Les télécommunications, supportées par des faisceaux hertziens, permettent l'envoi en temps réel des données en sortie du Castor3 vers la Météopole de Toulouse.

Le traitement en temps réel de ces gigantesques flux de données requiert une ingénierie efficace décrite ci-après.

\section{Ingénierie de la plateforme logicielle radar Rhytmme}

Les données utilisées par la plateforme radar Rhytmme proviennent des radars polarimétriques en bande X (mont Vial, montagne Maurel, mont Colombis, puis Vars à compter de septembre 2013) et également des radars bande $C$ et $S$ du réseau opérationnel Aramis: radars polarimétriques (Collobrières, Montclar, Nîmes, Toulouse) et radars conventionnels (Aléria, Bollène, Opoul, Saint-Nizier, Sembadel).

Concernant les radars conventionnels, ce sont des données issues de prétraitements effectués sur les calculateurs Castor2 au pied de chacun des radars qui sont envoyées en temps réel vers la Météopole sous forme de réflectivité composite (c'est-à-dire multisite) et lames d'eau, dites « locales », c'est-à-dire pour chaque radar pris individuellement.

Concernant les radars polarimétriques, ce sont des données brutes encore dites «tour d'antenne » qui sont envoyées. Il s'agit des mesures effectuées lors d'une rotation complète du radar à un site donné (six à huit fichiers de mesures sont envoyés toutes les cinq minutes). L'affichage de ces données sur un graphe en deux dimensions, où le radar est situé au centre et les échos radar placés selon l'azimuth et la distance par rapport au centre, est une méthode classique de visualisation connue sous son sigle anglais PPI (plan position indicator). Les données de l'ensemble de ces radars représentent un volume de l'ordre de 10 Go par jour.

Le prétraitement des données des radars polarimétriques ainsi que leur transformation en réflectivité et lames d'eau par les algorithmes polarimétriques (voir Tabary et al., 2013b, dans ce numéro) sont effectués sur la Météopole. Enfin, à partir de ces réflectivités et lames d'eau locales, des cartes mosaïques de réflectivité et de lame d'eau sont réalisées en combinant les informations de l'ensemble des radars du réseau et couvrant ainsi largement le quart sud-est de la France. Ces cartes sont fournies toutes les cinq minutes, à une résolution spatiale kilométrique. Elles représentent un volume d'environ 500 Mo par jour.

Pour les problématiques pluviologiques et hydrologiques ciblées dans ce projet, la lame d'eau est la donnée élaborée clé qui est exploitée. L'ensemble des services développés à partir de celle-ci sont décrits ci-après.

\section{La plateforme de services}

Depuis plus d'une décennie, de plus en plus de pays ou régions soumis à une multitude de risques (naturels, industriels...) déploient des approches intégrées pour aider les autorités gestionnaires à la prise de décision. Ces approches visent souvent à rassembler de manière ergonomique des informations qui sont par nature disséminées. Concernant les plateformes 
s'attelant aux risques naturels similaires à ceux visés dans Rhytmme, on peut citer les travaux de Kappes et al. (2012), Petzold et al. (2012), Giuliani et Peduzzi (2011), Heil et al. (2010) et Bründl et al. (2009). En général, il s'agit de faciliter le partage ou l'accès aux données en temps réel via les technologies de l'Internet dans des formats normés compatibles entre différentes plateformes.

\section{Son contenu}

Les lames d'eau de la plateforme logicielle radar nourrissent en temps réel une autre plateforme logicielle, appelée plateforme de services. Cette plateforme met à disposition via une interface web différents types de cartes, détaillées ci-après, avec toute une panoplie de fonctionnalités (cinq jours de données en ligne, choix de fonds de carte, choix de l'affichage des couches et de leur niveau de transparence, contrôle des animations temporelles, jauge informative, possibilité de visualiser des situations passées par requête auprès de l'administrateur système...). Son emprise spatiale dépasse largement le quart sud-est de la France pour bien pouvoir apprécier le déplacement des phénomènes sur la zone d'intérêt.
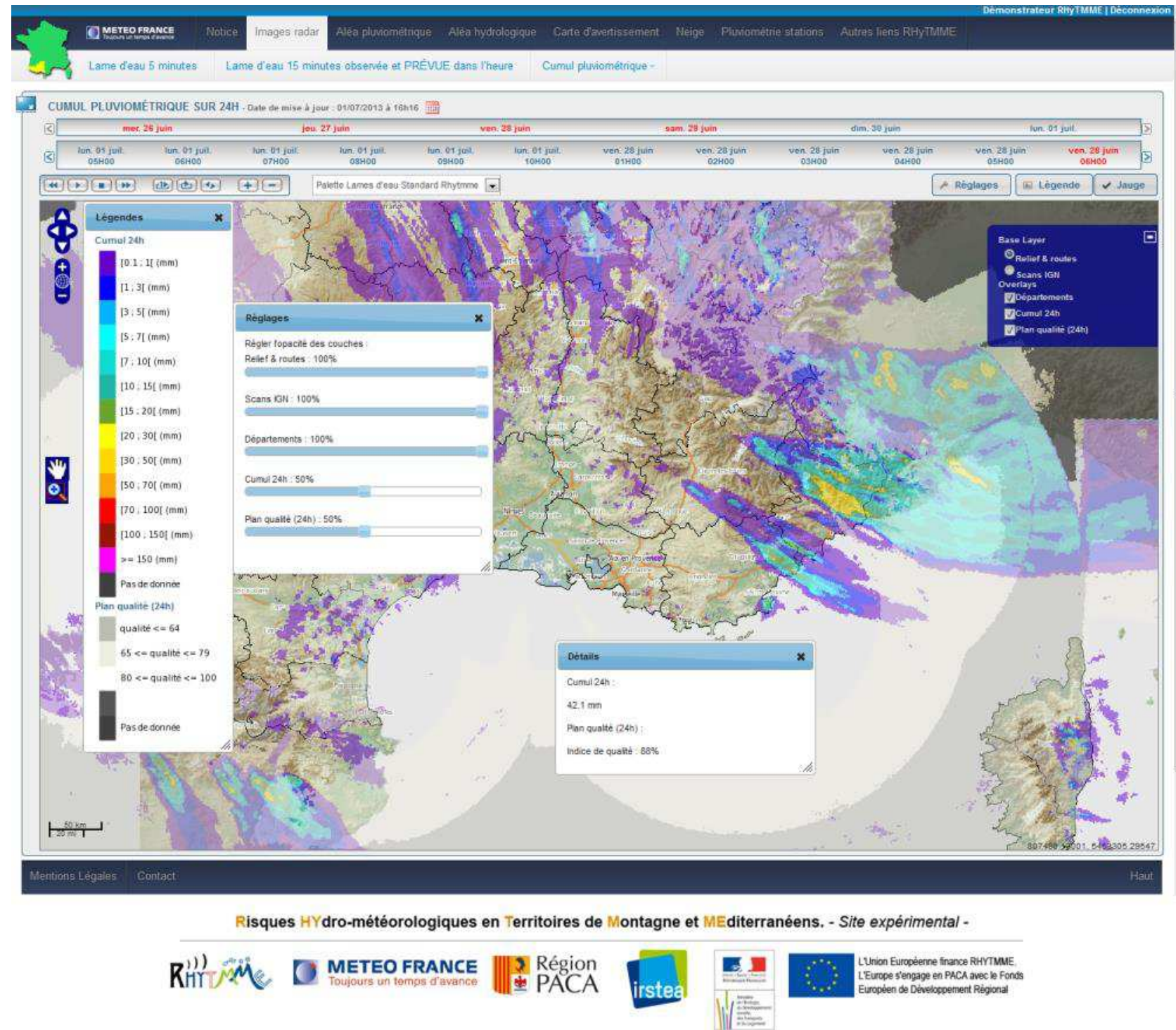

Figure 6. Vue générale de la plateforme de services affichant une carte de cumul de pluie sur une durée de $24 \mathrm{~h}$ estimé par le réseau radar Rhytmme le 28 juin 2013 à $6 \mathrm{~h}$ locales et les codes qualité radar associés en transparence. Les onglets des deux bandeaux supérieurs permettent de choisir le champ visualisé ; dessous, deux barres de navigation temporelle permettent de choisir jour et heure visualisés. Les différentes fenêtres pop-up affichées volontairement sur cet exemple permettent de contrôler les paramètres de visualisation ainsi que légende et jauge. 
Les lames d'eau sont cumulées sur différentes durées (de 5 min à 3 jours) avec calcul des codes qualité radar associés. Les cumuls 5 min sont produits toutes les 5 minutes, les cumuls sur les durées allant de 15 minutes à $12 \mathrm{~h}$ à la fréquence du quart d'heure et les cumuls de durée supérieure à la fréquence horaire (figure 7). Depuis peu ont été rajoutés trois cumuls de longue durée (semaine, mois, bimestre à fréquence quotidienne), utiles pour l'anticipation des mouvements de terrain.

Des cartes de pluies prévues dans l'heure sont générées par transport passif des pluies observées selon un déplacement calculé en comparant deux images radar consécutives (Juvanon du Vachat et al., 1995). Cette méthode fournit au pixel radar la valeur de précipitation prévue associée au quantile $50 \%$, mais aussi aux quantiles 20 et $80 \%$ donnant ainsi une indication de la confiance associée au quantile médian. Elle a ses limites, car les précipitations sont considérées d'intensité constante dans leur déplacement et l'influence du relief ainsi que la dynamique non linéaire des phénomènes météorologiques (orages, par exemple) ne sont pas prises en compte ; c'est pourquoi l'échéance de prévision n'est que d'une heure.

Les cumuls de pluie observés sur les durées de 1 heure à 3 jours sont confrontés en temps réel à des valeurs statistiques de pluies de durées de retour 2, 10 et 50 ans selon la méthode Aiga (Adaptation d'information géographique pour l'alerte en crue ; Lavabre et Grégoris, 2005) développée conjointement par l'Irstea et Météo-France. Aiga permet de définir le caractère plus ou moins exceptionnel des précipitations et de quantifier l'aléa pluviologique.

Les lames d'eau alimentent également un modèle hydrologique distribué de l'Irstea (Javelle et al., 2010), c'est-à-dire qui prend en compte la variabilité spatiale des processus qui ont lieu sur le bassin versant, comme par exemple la variabilité spatiale des pluies à l'échelle du $\mathrm{km}^{2}$, capturée par les mesures radar. Ce modèle hydrologique est adapté à des bassins versants de superficie supérieure à $10-20 \mathrm{~km}^{2}$ et à des cours d'eau non jaugés, c'est-à-dire ne disposant pas de station de mesure temps réel des débits (ce qui est le cas de la grande majorité des cours d'eau en zone méditerranéenne).

De façon similaire à l'aléa pluviologique, en confrontant les débits calculés en temps réel à l'exutoire des cours d'eau aux valeurs statistiques de débits de crue de période de retour 2, 10 et 50 ans, Aiga informe quant au caractère exceptionnel des débits modélisés des cours d'eau et quantifie l'aléa crue en ne prenant en compte que les pluies observées (et non les pluies prévues) (voir figure 11). Les valeurs statistiques de durées de retour de pluie et de débit sont issues de la méthode Shyreg développée par l'Irstea, en association avec Météo-France pour la partie pluviométrie (Organde et al., 2013 ; Arnaud et al., 2008).

En complément à la cartographie des aléas hydrologiques (voir figure 11), pour chaque couple bassin versant/cours d'eau, l'accès en temps réel aux hyétogramme (évolution temporelle des quantités de pluie) et hydrogramme de crue (évolution temporelle des débits modélisés) donne une information plus précise sur l'évolution de la situation au regard des différents seuils de crue tabulés (figure 7). 
Author-produced version of the article published in La Meteorologie, 2013, 83, 8, pp. 69-79

Original publication available at http://documents.irevues.inist.fr/handle/2042/52056

doi : $10.4267 / 2042 / 52056$

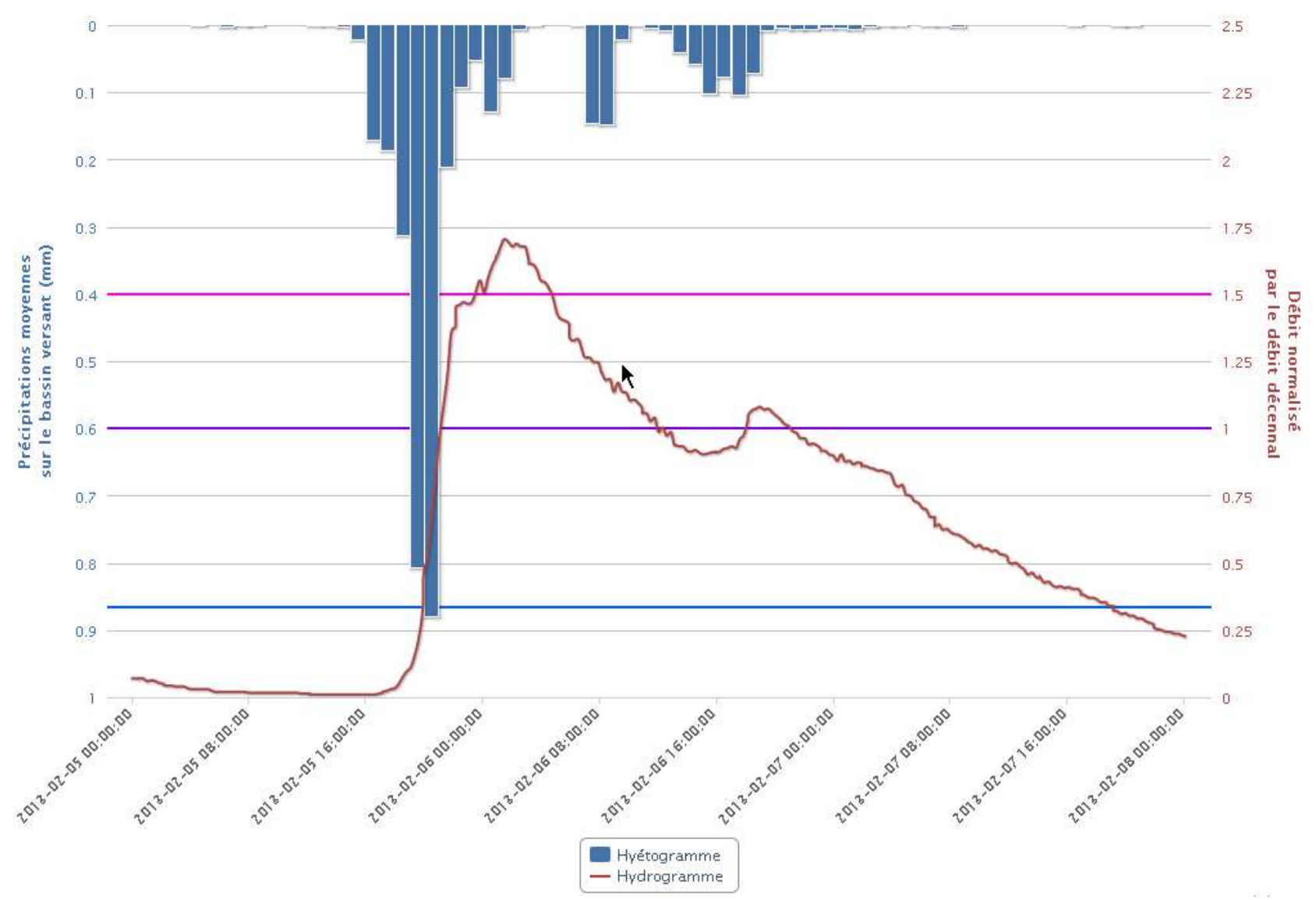

Figure 7. Hyétogramme (évolution temporelle des quantités de pluie) et hydrogramme (évolution temporelle des débits) en temps réel visualisables sur la plateforme de services. L'hydrogramme est normalisé par le débit de crue décennal, avec report des seuils de période de retour ( 2 ans en bleu foncé, 10 ans en violet et 50 ans en rose) des débits de crue (modèle Aiga hydro).

À partir de la qualification des aléas par Aiga, des avertissements par courriel sont constitués à l'échelle des communes pour ce qui est de l'aléa pluviologique et à l'échelle des tronçons de cours d'eau pour ce qui est de l'aléa hydrologique. Deux niveaux existent : aléa pluies intenses ou très intenses et aléa de crue importante ou très importante. Ces avertissements sont envoyés aux utilisateurs qui le souhaitent et sont aussi cartographiés sur la plateforme de services. À terme, ces avertissements pourront aussi être relayés par SMS ou appel téléphonique si souhaité.

Très prochainement seront intégrées à la plateforme des cartes à grande échelle (résolution de quelques hectares) de sensibilité à l'aléa torrentiel (figure 8) et aux aléas de mouvements de terrain (glissements et chutes de blocs), sur les trois départements alpins pour la première (Bertrand et al., 2013) et l'ensemble de la région Provence-Alpes-Côte d'Azur pour les secondes (Batista et al., 2013a; Batista et al., 2013b). Ces cartes pourront être croisées en temps réel avec les cartes d'aléas pluviologiques (à différentes profondeurs de temps) et hydrologiques. À terme, des seuils de risque de déclenchement de phénomènes seront ajoutés, suite aux résultats d'analyses bibliographiques en cours à propos de la phénoménologie des laves torrentielles (Bacchini et Zannoni, 2003) et des mouvements de terrain (Sandersen et al., 1997). 


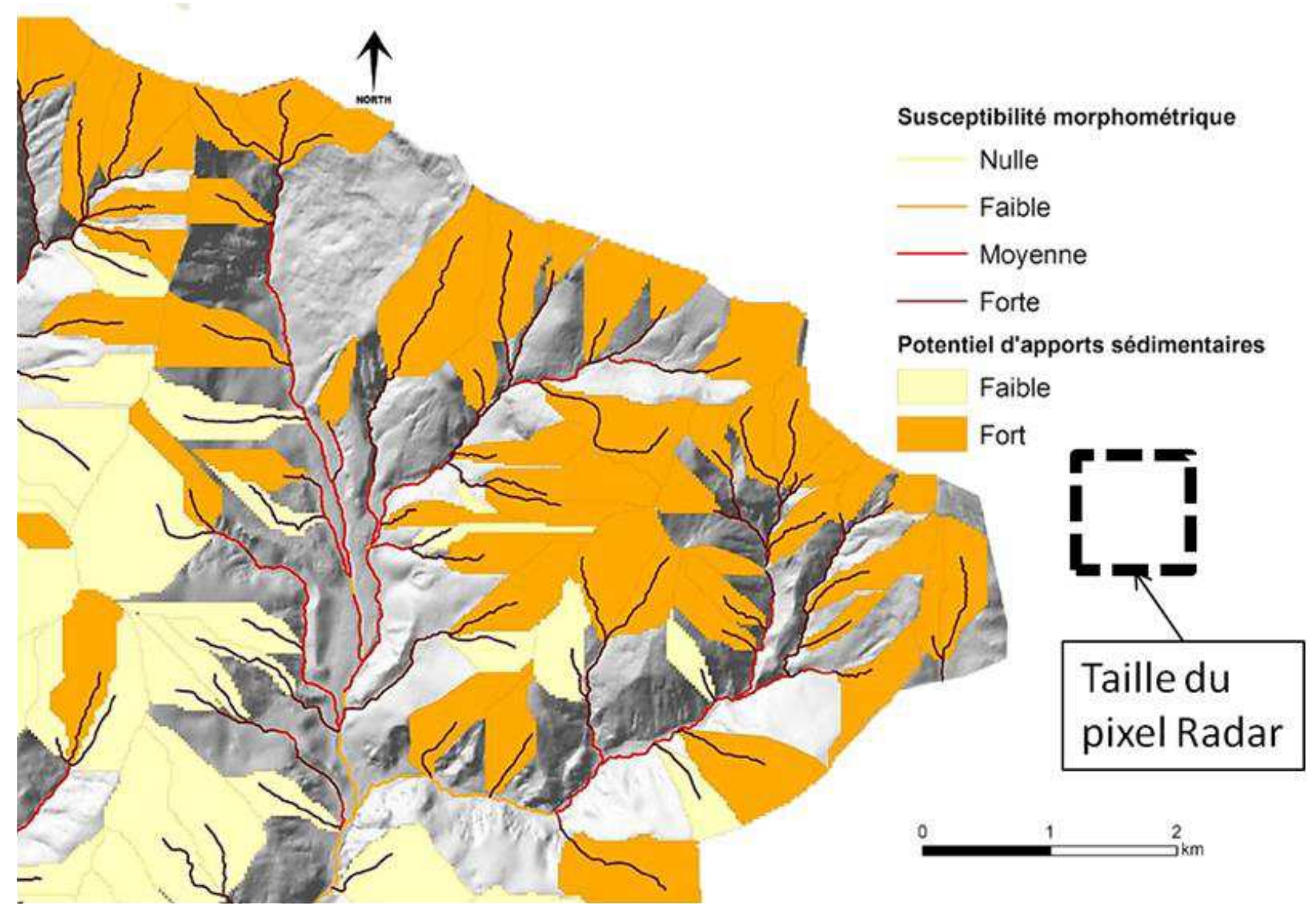

Figure 8. Carte de susceptibilité morphométrique à l'aléa torrentiel dans le Haut Var et potentiel d'apports sédimentaires des bassins versants.

Enfin, les mesures effectuées par le réseau de pluviomètres au sol sont aussi disponibles sur la plateforme. Elles permettent aux utilisateurs de vérifier localement la cohérence des mesures radar avec celles des pluviomètres ainsi que de documenter les événements observés.

Accessible depuis une plateforme internet et relayé sous forme d'avertissement courriel, l'ensemble de ces services est testé par un groupe d'expérimentateurs.

\section{Test des services par un groupe d'expérimentateurs}

L'une des originalités du projet Rhytmme est le choix de mettre à la disposition d'un groupe d'expérimentateurs, représentatifs des gestionnaires du risque en montagne, les nouveaux services et fonctionnalités de la plateforme de services dans un délai très bref après leur phase de recherche-développement. La première version de cette plateforme a ainsi été livrée en novembre 2011 ; l'accès se fait par un identifiant et un mot de passe génériques.

Le groupe d'expérimentateurs compte à ce jour une centaine de contributeurs relevant notamment des services déconcentrés de l'État, des conseils généraux, de communes ou de communautés de communes, de syndicats de rivière, d'un parc naturel et de sociétés industrielles publiques ou privées. Les expérimentateurs ont pour mission de : se connecter aussi souvent que possible à la plateforme de services web lorsque des événements pluvieux sont annoncés ou en cours sur leur territoire d'intérêt respectif ; recenser les difficultés rencontrées en terme de lisibilité et d'interprétation des informations affichées ou transmises par la plateforme ; évaluer la pertinence des informations transmises en regard d'observations ou de témoignages remontant du terrain ; exprimer le besoin d'information supplémentaire et/ou de modification de la présentation des résultats. À titre d'exemples, nous revenons ci-après sur deux événements hydrométéorologiques remarquables de l'année 2012 dans les Alpes du Sud vus de la plateforme de services. 
Orage de printemps dans le Serrois le 24 mai 2012

Un violent orage de pluie et de grêle a eu lieu dans les environs de la ville de Serres (HautesAlpes, environ $30 \mathrm{~km}$ au sud-ouest de Gap) le 24 mai 2012 en fin d'après-midi. Les ruissellements et ravinements qui en ont résulté, en dépit de la brièveté du phénomène, ont provoqué d'importants dégâts dont une demi-douzaine de débordements de torrent et un glissement de remblai sur la voie ferrée reliant Aix-en-Provence et Gap, au sud de Serres, ayant conduit la SNCF à stopper le trafic ferroviaire vers $18 \mathrm{~h}$ (figure 9).

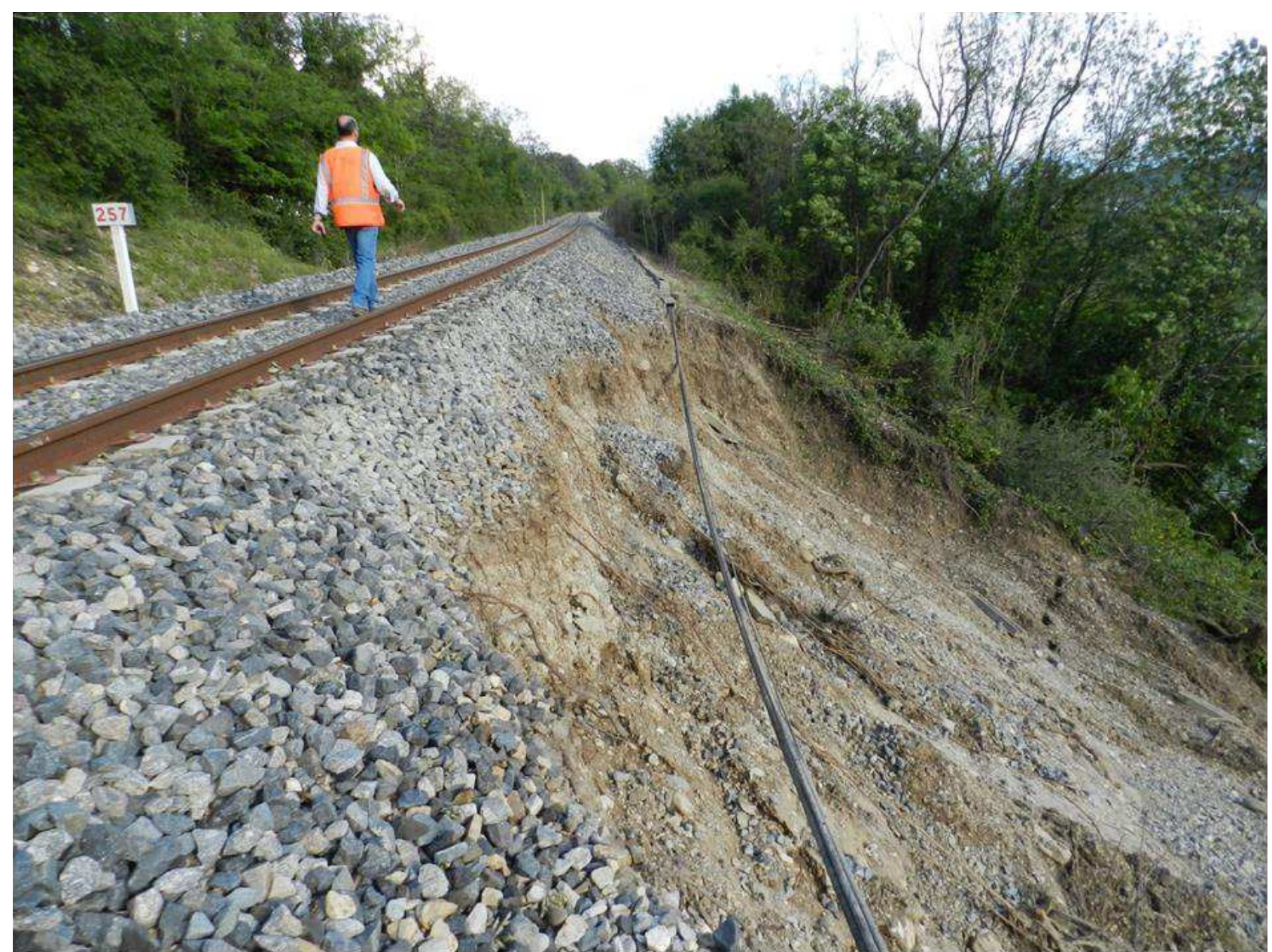

Figure 9. Glissement d'environ $5000 \mathrm{~m}^{3}$ du remblai de la voie ferrée au sud de Serres, constaté le 24 mai 2012 à 18 h30 (photo (C) Patrice Mériaux, Irstea, 26 mai 2012).

Le suivi de cet événement par la plateforme Rhytmme montre que les précipitations les plus importantes se sont produites sur un secteur très localisé entre $17 \mathrm{~h} 30$ et $18 \mathrm{~h} 45$. Sur une étroite bande d'environ $15 \mathrm{~km}$ de long sur $5 \mathrm{~km}$ de large (figure 10), la lame d'eau radar est fortement surestimée (cumul de plus de $150 \mathrm{~mm}$ en $3 \mathrm{~h}$ ), conséquence de l'occurrence avérée de grêle (témoignages relatés par la presse locale, photos à l'appui). Les estimations de lame d'eau radar (50-70 $\mathrm{mm}$ ) à la périphérie de cette zone sont en accord avec les mesures au sol (62 mm sur la totalité du phénomène au pluviomètre de Valdrôme, Haute Drôme). 


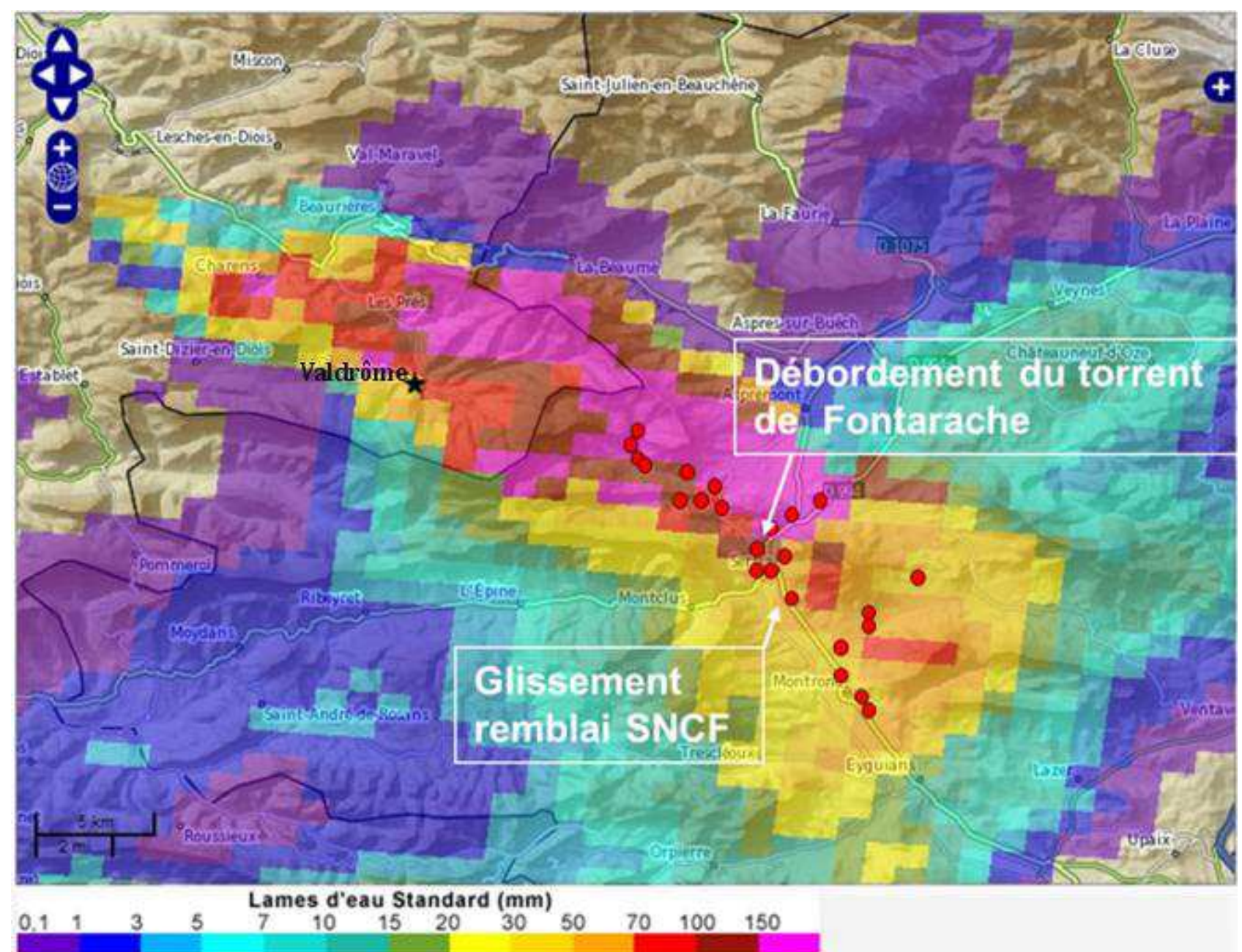

Figure 10. Carte de cumul de pluie estimé par le réseau de radars lors de l'orage du 24 mai 2012 à 19 h locales sur une durée de $3 \mathrm{~h}$ dans les environs de Serres (Hautes-Alpes, environ $30 \mathrm{~km}$ au sud-ouest de Gap). Les pixels de $1 \mathrm{~km}^{2}$, échelle spatiale à laquelle les cumuls de pluie sont estimés, sont bien visibles. Les lieux de dégât recensés par le service départemental de restauration des terrains en montagne des Hautes-Alpes sont symbolisés par des points rouges. L'étoile noire situe le village de Valdrôme où un pluviomètre a enregistré un cumul de pluie de $62 \mathrm{~mm}$ sur la totalité de l'événement.

Le service départemental de restaurations des terrains en montagne (RTM-ONF) des HautesAlpes, instructeur des dossiers de déclaration de catastrophe naturelle et membre du groupe d'expérimentateurs, a procédé à un recensement exhaustif de tous les dégâts déplorés au sol lors de l'événement. Leur localisation (figure 10) montre qu'ils se situent tous dans une large zone de lame d'eau radar supérieure à $50 \mathrm{~mm}$, au moins en ce qui concerne le département des Hautes-Alpes (le département de la Drôme, concerné par la partie nord-ouest du noyau important de précipitations, n'a pas été sondé). L'absence de dégâts particuliers à l'intérieur de la bande de lame d'eau surestimée fait penser que la surestimation liée à la grêle y est au moins d'un facteur 2 à 3.

Il est à noter que les débordements de torrents ou ravins ont concerné des cours d'eau de bassin versant de superficie inférieure à $10 \mathrm{~km}^{2}$, donc trop petits pour être traités dans la version actuelle du modèle hydrologique utilisé dans Aiga. Or, les victimes de crues éclair sur la route sont souvent situées sur de tout petits bassins (Ruin et al., 2008). Dans une prochaine version de la plateforme, la base de données des bassins versants sera étendue à ceux de 
Author-produced version of the article published in La Meteorologie, 2013, 83, 8, pp. 69-79

Original publication available at http://documents.irevues.inist.fr/handle/2042/52056

doi : $10.4267 / 2042 / 52056$

superficie comprise entre 5 et $10 \mathrm{~km}^{2}$, ce qui est intéressant au vu de la dangerosité des très petits bassins versants ${ }^{5}$, notamment en montagne et/ou vis-à-vis du réseau routier.

Ce même 24 mai, la SNCF, également expérimentatrice de Rhytmme, a été témoin direct de l'événement en gare de Serres. Le chef de gare, voyant les quais submergés par les ruissellements intenses, a fort heureusement fait stopper les trains vers $18 \mathrm{~h}$, le glissement de remblai plus au sud n'ayant été découvert qu'un peu plus tard vers $18 \mathrm{~h} 30$. Si cet épisode avait touché, plus haut dans la montagne, une portion de voie isolée, le chef de gare 6 ou le responsable local du trafic aurait pu, à l'examen d'images Rhytmme qualifiant en temps réel l'intensité du phénomène hydrométéorologique quel que soit son lieu d'occurrence, faire stopper ou ralentir les trains dans le secteur concerné, jusqu'à ce qu'une équipe de surveillance envoyée spécialement « sur zone » puisse vérifier l'existence éventuelle de dégâts ou de menaces sur la voie. C'est clairement ce type de service qu'attendent les opérateurs ferroviaires (SNCF et Chemins de fer de Provence) pour gérer la sécurité des centaines de kilomètres de voies ferrées régionales qui traversent les reliefs escarpés des Alpes du Sud.

\section{Épisode pluvio-orageux des 4-5 novembre 2012 dans le Haut Verdon et la Haute Bléone}

Cet événement nous permet d'illustrer cette fois-ci les services d'anticipation des crues (cartes d'« aléa hydrologique ») disponibles dans Rhytmme. L'examen des images Rhytmme montre que les précipitations ont débuté vers $13 \mathrm{~h}$ le 4 novembre 2012 en s'intensifiant à partir de $17 \mathrm{~h}$ et se sont achevées vers $2 \mathrm{~h}$ le 5 novembre. Dans la zone géographique affectée (nord-est des Alpes-de-Haute-Provence et nord des Alpes-Maritimes), les premières mises en garde hydrologiques (débit modélisé dépassant le seuil de période de retour de 2 ans) apparaissent à $18 \mathrm{~h} 45$, les premiers avertissements de crue importante (période de retour supérieure à 10 ans) à $19 \mathrm{~h} 30$, puis un premier avertissement de crue très importante (période de retour supérieure à 50 ans) à $20 \mathrm{~h} 30$ et sept rivières torrentielles en avertissement de crue très importante à $23 \mathrm{~h} 30$ (figure 11).

\footnotetext{
${ }^{5}$ L'intérêt d'intégrer dans Aiga des superficies encore plus petites est à discuter car, tant que le modèle pluiedébit fonctionne avec des pluies mesurées (et non prévues), le délai d'anticipation des crues à l'exutoire reste de l'ordre de grandeur du temps de concentration du bassin, qui descend sous le quart d'heure pour les plus petits d'entre eux.

${ }^{6}$ On précise que les chemins de fer de montagne sont généralement à voie unique et que les principales gares constituent les points d'arrêt pour le croisement des convois. Lors de ces arrêts, il revient au chef de gare local de donner ou non l'autorisation de départ des trains.
} 


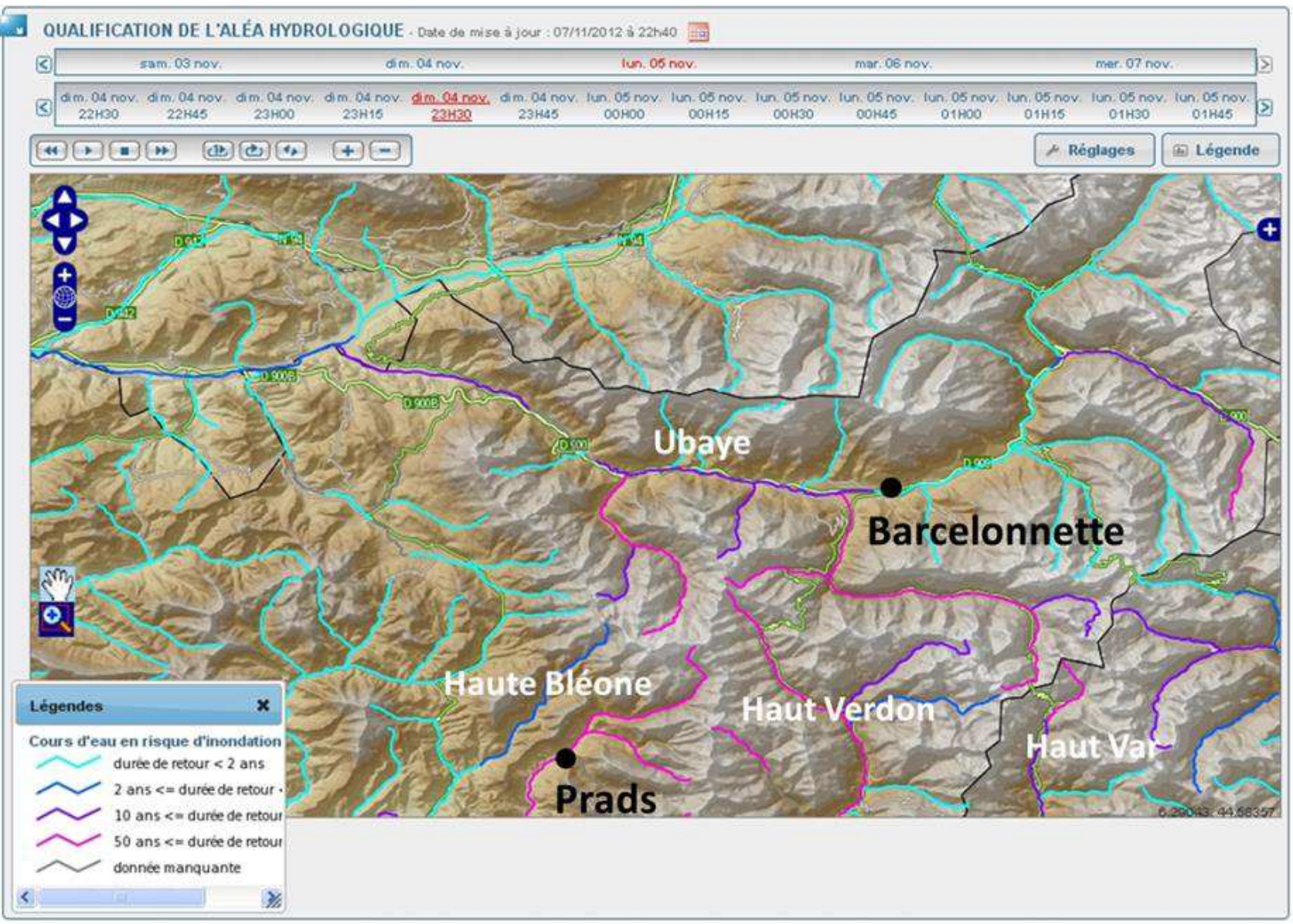

Figure 11. Carte de qualification de l'aléa hydrologique dans la région de Barcelonnette (nord-est des Alpes-deHaute-Provence) le 4 novembre 2012 à 23h30. La distance horizontale de Prads à Barcelonnette est d'environ $25 \mathrm{~km}$. La nomenclature des niveaux de gravité des événements est la suivante :

- bleu clair pour les débits estimés inférieurs à la période de retour de 2 ans des quantiles de référence ;

- bleu foncé lorsque les débits estimés dépassent la période de retour de 2 ans des quantiles de référence ;

- violet lorsque les débits dépassent la période de retour de 10 ans des quantiles de référence ;

- rose lorsque les débits dépassent la période de retour de 50 ans des quantiles de référence.

Les expérimentateurs (Service RTM 04 et Syndicat de la Bléone, en particulier) ont fait remonter dans les jours qui ont suivi des fiches de déclaration de dégâts qui confirment la pertinence géographique des avertissements de crue (figure 12), avec toutefois des signatures sur le terrain paraissant correspondre à des intensités moindres que celles annoncées par le module hydrologique déployé dans Rhytmme. 


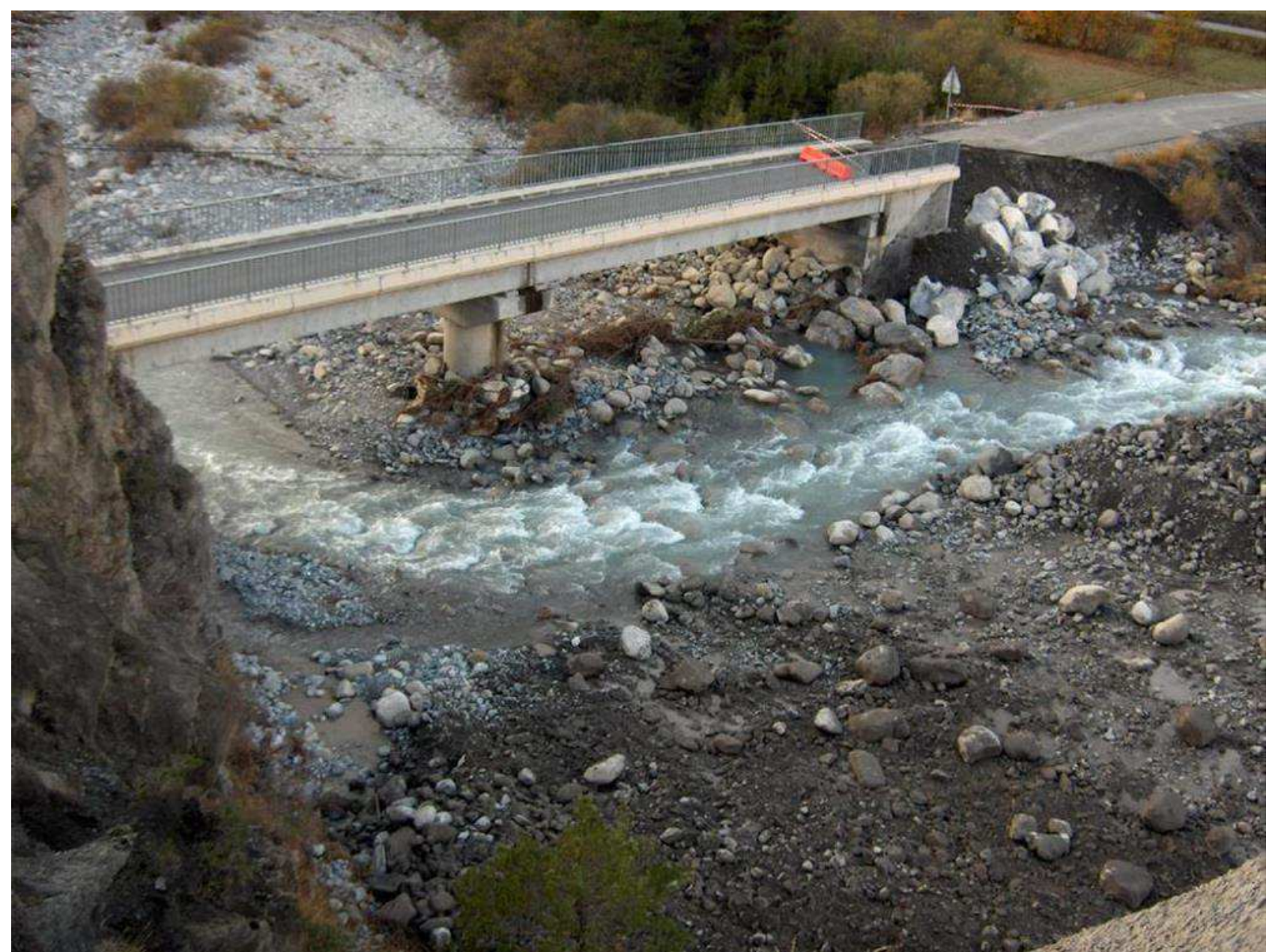

Figure 12. Passerelle contournée sur la Bléone à Prads, après la crue torrentielle des 4 et 5 novembre 2012 (photo@ Jean-Yves Sabinen, Onema, 06/11/2012).

Un deuxième événement hydrométéorologique s'est produit dans le bassin versant de la Bléone quelques jours après (les 10 et 11 novembre), provoquant a priori de nouveaux dégâts ou des aggravations d'érosion au bord des torrents. Certains cours d'eau se sont affichés en avertissement intense sur la plateforme de services. Il n'est alors pas évident de rattacher les désordres constatés au sol à l'événement du 4-5 novembre ou à celui du 10-11 novembre, sauf à avoir effectué une inspection des torrents entre les deux épisodes. Ceci illustre une difficulté pour le suivi expérimental de la plateforme dans les zones reculées de montagne.

\section{Bilan des retours d'expérience}

Les premiers retours d'expérience issus de l'analyse des événements récents tels que vus par la plateforme Rhytmme montrent à la fois l'intérêt de l'outil et les travaux d'amélioration qu'il reste à accomplir.

Les impressions générales suivantes se dégagent du groupe d'expérimentateurs :

- les cartes affichées sur la plateforme de services permettent d'expliquer a posteriori des événements survenus ; vice versa, les avertissements guident sur les lieux où rechercher des événements qui n'ont pas forcément eu un grand retentissement, faute de témoins directs ; - l'anticipation par Rhytmme des crues soudaines estivales pour les bassins versants de montagne de plusieurs dizaines de kilomètres carrés apparaît informative, notamment pour la gestion de la sécurité des nombreux campings qui bordent les cours d'eau concernés ;

- même si l'anticipation du paroxysme des événements est encore faible pour les très petits bassins versants, le suivi en temps réel de la dynamique des situations couplé à une excellente 
connaissance locale du terrain permet aux expérimentateurs de discriminer certaines situations génératrices de désordres.

Le groupe d'expérimentateurs reste plus que jamais mobilisé dans la perspective prochaine de la mise à disposition de services d'avertissement propres aux aléas de montagne : crues torrentielles et mouvements de terrain. Les expérimentations vont ainsi se poursuivre jusqu'à fin 2014 afin de recueillir un maximum d'informations de retour d'expérience.

\section{Conclusion}

Cinq années après le lancement du projet Rhytmme, un réseau de radars Doppler polarimétriques en bande $X$ (trois radars installés) complétant la couverture du réseau Aramis a été déployé sur les Alpes du Sud. Les algorithmes de traitement de ses mesures, spécifiques aux données polarimétriques et adaptés aux trois bandes $S, C$ et $X$, ont été développés et tournent en temps réel pour délivrer notamment des quantités de pluies observées, typiquement à l'échelle de $1 \mathrm{~km}^{2}$ et au pas de temps de $5 \mathrm{~min}$. Ces pluies alimentent elles-mêmes d'autres logiciels qui produisent des cartes hydrométéorologiques et des avertissements renseignant sur la probabilité d'occurrence d'aléa de type crue, sur l'ensemble du réseau hydrographique de la région Provence-Alpes-Côte d'Azur couvert par le réseau radar. L'ensemble de ces informations est consultable en temps réel sur une plateforme de services accessible par internet et qui est actuellement testée par un groupe d'utilisateurs expérimentés dans la gestion des risques naturels. Les retours d'expérience recensés à ce jour indiquent une utilité des informations de la plateforme pour le suivi en direct des situations potentiellement dangereuses même si le délai d'anticipation est souvent court. La plateforme continue à s'enrichir avec l'arrivée imminente de cartographies des zones susceptibles de produire des laves torrentielles, des glissements de terrain, des chutes de blocs, qui, couplées à la qualification des aléas pluviologiques et hydrologiques, permettront de mieux apprécier localement le risque d'occurrence d'un aléa de ce type.

Par ailleurs, un modèle économique permettant de pérenniser l'ensemble « réseau de radars et plateforme de services" est en réflexion; il définira lesquelles des informations actuellement délivrées expérimentalement continueront à l'être dans un cadre opérationnel et dans quelles conditions une fois le projet Rhytmme fini.

Les innovations technologiques et algorithmiques issues de ce projet pilote ont vocation à bénéficier à d'autres régions vulnérables, notamment en zones montagneuses, ainsi qu'à d'autres projets visant à prévenir les crues soudaines. C'est par exemple le cas de ce qui est envisagé à courte échéance dans la région grenobloise avec l'installation d'un radar Doppler polarimétrique en bande X, projetée fin 2014, sur le sommet du Moucherotte dans le Vercors.

\section{Remerciements}

Les auteurs et leur organisme tiennent à remercier la Région Provence-Alpes-Côte d'Azur, l'Union européenne (Feder) et le ministère en charge de l'Écologie pour leur important soutien financier au projet Rhytmme, au titre du contrat de projets État-Région 2007-2013, ainsi que les nombreux organismes et personnes impliqués dans le projet (plus de 100 personnes représentant plus de 50 équivalent temps plein). Les auteurs remercient également les deux relecteurs pour leurs remarques constructives qui ont permis d'améliorer significativement l'article. 
Author-produced version of the article published in La Meteorologie, 2013, 83, 8, pp. 69-79

Original publication available at http://documents.irevues.inist.fr/handle/2042/52056

doi : $10.4267 / 2042 / 52056$

\section{Bibliographie}

Andrieu H. et J.D. Creutin, 1995 : Identification of vertical profiles of radar reflectivity for hydrological applications using an inverse method. 1. Formulation. J. Appl. Meteorol., 34, 225239.

Andrieu H., G. Delrieu et J.D. Creutin, 1995 : Identification of vertical profiles of radar reflectivity for hydrological applications using an inverse method. 2. Sensitivity analysis and case-study. J. Appl. Meteorol., 34, 240-259.

Arnaud P., J. Lavabre, B. Sol et C. Desouches, 2008 : Régionalisation d'un générateur de pluies horaires sur la France métropolitaine pour la connaissance de l'aléa pluviographique, Hydrological Sci. J., 53, 34-47.

Bacchini M. et A. Zannoni, 2003 : Relations between rainfall and triggering of debris flow: case study of Cancia (Dolomites, Northeastern Italy). Nat. Haz. Earth System Sci., 3,71-79.

Batista D., P. Azémard et M. Boutry, 2013a : Projet RHYTMME : prévision de l'aléa glissement de terrain et analyse statistique des facteurs de prédisposition par l'outil SIG, sur la région Provence-Alpes-Côte d'Azur (PACA). Journées Aléas gravitaires (Grenoble, France), 17-18 septembre 2013.

Batista D., P. Azémard, A.-C. Rougé, M. Dumalin et C. Rault, 2013b : Prévision de l'aléa chute de blocs, analyse statistique des facteurs de prédisposition et des critères de déclenchement sur la région Provence-Alpes-Côte d'Azur (PACA). Journées Aléas gravitaires (Grenoble, France), 17-18 septembre 2013.

Bertrand M., F. Liébault et H. Piégay, 2013 : Debris-flow susceptibility of upland catchments. Nat. Haz., 67, 497-511.

Bringi V.N. et V. Chandrasekar, 2001 : Polarimetric Doppler Weather Radar: Principles and Applications. Cambridge University Press.

Bründl M., B. Krummenacher et H. Merz, 2009 : Decision making tools for natural hazard risk management - Examples from Switzerland, in: Safety, Reliability and Risk Analysis - Theory, Methods and Applications, edited by Martorell S., Soares C.G., et Barnett J., CRC Press/Balkema, Leiden, 4, 2773-2779.

Cremonini R., P. Tabary, J. Sugier, M. Frech, P.P. Alberoni, L. Baldini, A Huuskonen et A. Vocino, 2012: Evaluation of X-band radar technologies within Opera 3 project. 7th European Conference on Radar in Meteorology and Hydrology (Toulouse, France), 24-29 June 2012.

Delrieu G., H. Andrieu et J.D. Creutin, 2000 : Quantification of path-integrated attenuation for $\mathrm{X}$ - and C-band weather radar systems operating in Mediterranean heavy rainfall. J. Appl. Meteorol., 39, 840-850.

Delrieu G., S. Serrar, E. Guardo et J.D. Creutin, 1999a : Rain measurement in hilly terrain with X-band weather radar systems: Accuracy of path-integrated attenuation estimates derived from mountain returns. J. Atmos. Oceanic Technol., 16, 405-416.

Delrieu G., L. Hucke et J.D. Creutin, 1999b: Attenuation in rain for X- and C-band weather radar systems: Sensitivity with respect to the drop size distribution. J. Appl. Meteorol., 38, 5768.

Delrieu G., S. Caoudal et J.D. Creutin, 1997 : Feasibility of using mountain return for the correction of ground-based X-band weather radar data. J. Atmos. Oceanic Technol., 14, 368385.

Delrieu G. et J.D. Creutin, 1991: Weather radar and urban hydrology: advantages and limitations of X-band light configuration systems. Atmos. Res., 27, 159-168.

Diss S., G. Scialom, E. Le Bouar, J. Testud, J. Lavabre et J.L. Cheze, 2006: FRAMEA: an experimental campaign for severe storm survey and flood warning, associating an x-band weather radar and a discharge hydrological model, Proceedings of ERAD 2006, 4th European conference on radar in meteorology and hydrology (Barcelona, Espagne), 18-22 September 2006, 429-431. 
Author-produced version of the article published in La Meteorologie, 2013, 83, 8, pp. 69-79

Original publication available at http://documents.irevues.inist.fr/handle/2042/52056

doi : $10.4267 / 2042 / 52056$

Diss S., J. Testud, J. Lavabre, P. Ribstein, E. Moreau et J. Parent du Châtelet, 2009 : Ability of a dual polarized X-band radar to estimate rainfall. Adv. Water Res., 32, 975-985.

Dupouyet J.-P., 1983 : Le radar et la pluie. Application à l'annonce et à la prévision des crues dans les bassins critiques de la Vézère et de la Corrèze. La Houille Blanche, 5/6, 387-392.

Feiertag N., K. Lengfeld, M. Clemens et F. Ament, 2012: Precipitation and attenuation estimates from a high resolution weather radar network - Development of retrievals for a radar network retrievals for a radar network. 7th European Conference on Radar in Meteorology and Hydrology (Toulouse, France), 24-29 June 2012.

Frasier S. J., F. Kabeche, J. Fuigeras i Ventura, H. Al-Sakka, P. Tabary, J. Beck et O. Bousquet, 2013 : In-place estimation of wet radome attenuation at X band. J. Atmos. Oceanic Technol., 30, 917-928.

Germann U., M. Gabella, M. Boscacci, I. Sideris, M. Sartori, A. Hering, L. Clementi et M. Sassi, 2012: Next generation radar precipitation measurement in the Swiss Alps: strategy and first results. 7th European Conference on Radar in Meteorology and Hydrology (Toulouse, France), 24-29 June 2012.

Giuliani G. et P. Peduzzi, 2011 : The PREVIEW Global Risk Data Platform: a geoportal to serve and share global data on risk to natural hazards. Nat. Haz. Earth System Sci., 11, 53-66.

Heil B., I. Petzold, H. Romang et J. Hess, 2010 : The common information platform for natural hazards in Switzerland. Nat. Haz., doi: 10.1007/s11069-010-9606-6.

Javelle P., C. Fouchier, P. Arnaud et J. Lavabre, 2010 : Flash flood warning at ungauged locations using radar rainfall and antecedent soil moisture estimations. J. Hydrol., 394, 267274.

Juvanon du Vachat R., P. Thomas, E. Bocrie, G. Monceau, P. Cosentino, S. Sénési, D. Tzanos et J.L. Boichard, 1995: The precipitation nowcast scheme in the Aspic project. Proc. Second European Conf. on Applications of Meteorology, Toulouse, France, 29-32.

Kaltenboeck R., 2012: New generation of dual polarized weather radars in Austria. 7th European Conference on Radar in Meteorology and Hydrology (Toulouse, France), 24-29 June 2012.

Kappes M.S., K. Gruber, S. Frigerio, R. Bell, M. Keiler et T. Glade, 2012: The MultiRISK platform: The technical concept and application of a regional-scale multihazard exposure analysis tool. Geomorphology, 151, 139-155.

Lavabre J. et Y. Grégoris, 2005 : AIGA: un dispositif d'alerte des crues sur l'ensemble du réseau hydrographique. Ingénieries E A T, 44, 3-12.

Le Bouar E., J. Testud et T.D. Keenan, 2001 : Validation of the rain profiling algorithm "ZPHI" from the C-band polarimetric weather radar in Darwin. J. Atmos. Oceanic Technol., 18, 18191837.

Maesaka T., M. Maki, K. Iwanami, S. Tsuchiya, K. Kieda et A. Hoshi, 2011 : Operational Rainfall Estimation by X-band MP Radar Network in MLIT, Japan. Proc. of 35th AMS Int. Conf. on Radar Meteorology, Pittsburgh PA.

McLaughlin D., D., Pepyne, V. Chandrasekar, B. Philips, J. Kurose, M., Zink, K. Droegemeier, S. Cruz-Pol, F. Junyent, J. Brotzge, D. Westbrook, N. Bharadwaj, Y.T. Wang, E. Lyons, K. Hondl, Y.X. Liu, E. Knapp, M. Xue, A. Hopf, K. Kloesel, A. DeFonzo, P. Kollias, K. Brewste, R. Contreras, B. Dolan, T. Djaferis, E. Insanic, S. Frasier et F. Carr, 2009: Short-wavelength technology and the potential for distributed networks of small radar systems. Bull. Amer. Meteorol. Soc., 90, 17971817.

Mériaux P., S. Westrelin, J.-L. Chèze, P. Tabary, P. Javelle et D. Defrance, 2011 : Projet RHYTMME: Déploiement d'un réseau de radars dédié à la gestion des risques hydrométéorologiques en territoires montagneux méditerranéens. Colloque SHF «Eaux en montagne », Lyon, France, 16-17 mars 2011. 
Author-produced version of the article published in La Meteorologie, 2013, 83, 8, pp. 69-79

Original publication available at http://documents.irevues.inist.fr/handle/2042/52056

doi : $10.4267 / 2042 / 52056$

Organde D., P. Arnaud, J. A. Fine, C. Fouchier, N. Folton et J. Lavabre, 2013 : Régionalisation d'une méthode de prédétermination de crue sur l'ensemble du territoire français : La méthode SHYREG. Revue des Sciences de l'Eau, 26, 65-78.

Palumbo R., E. Knapp, K. Wood et D. McLaughlin, 2012: Deployment Considerations and Hardware Technologies for Realizing X-Band Radar Networks. 7th European Conference on Radar in Meteorology and Hydrology (Toulouse, France), 24-29 June 2012.

Pellarin T., G. Delrieu, G.M. Saulnier, H. Andrieu, B. Vignal et J.D. Creutin, 2002 : Hydrologic visibility of weather radar systems operating in mountainous regions: Case study for the Ardeche Catchment (France). J. Hydrometeorol., 3, 539-555.

Pellarin T., G. Delrieu et J.D. Creutin, 2000 : Hydrologic visibility of weather radars operating in high-mountainous regions: A case study for the Toce catchment (Italy) during the mesoscale alpine programme. Phys. Chem. Earth Part B-Hydrol. Oceans Atmos., 25, 953-957.

Petzold I., J. Hess, F. Schmid, M. Arpagaus et S. Steiner 2012 : Joint information platform for natural hazards in Switzerland. 12th Congress Interpraevent (Grenoble/France), 23-27 April 2012.

Ruin I., J.D. Creutin, S. Anquetin et C. Lutoff, 2008 : Human exposure to flash floods - Relation between flood parameters and human vulnerability during a storm of September 2002 in Southern France. J. Hydrol., 361, 199-213.

Sandersen F., S. Bakkehöi, E. Hestnes et K. Lied, 1997 : The influence of meteorological factors on the initiation of debris flows, rockfalls, rockslides and rockmass stability. PublikasjonNorges Geotekniske Institutt, 201, 97-114.

Sauvageot H., 1983 : Mesures hydrologiques par radar. La Houille Blanche, 5/6, 329-340.

Tabary P., C. Augros, J.-L. Champeaux, J.-L. Chèze, D. Faure, D. Idziorek, R. Lorandel, B. Urban et V. Vogt 2013a : Le réseau et les produits radars de Météo France. La Météorologie, 83, 15-27. Tabary P., B. Fradon et A.-A. Boumahmoud, 2013b : La polarimétrie radar à Météo-France. $L a$ Météorologie, 83, 59-67.

Tabary P., G. L'Henaff, P. Dupuy, J. Parent du Châtelet et J. Testud, 2008: Can we use polarimetric X-band radars for operational quantitative precipitation estimation in heavy rain regions? International Symposium on Weather Radar and Hydrology (Grenoble, France), 10 15 March 2008.

Testud J., 2002 : Le radar météorologique à diversité de polarisation : un outil prêt à entrer dans l'application opérationnelle. La Houille Blanche, 2, 46-49.

Testud J., E. Le Bouar, E. Obligis et M. Ali-Mehenni, 2000 : The rain profiling algorithm applied to polarimetric weather radar. J Atmos. Ocean Technol., 17, 332-356.

Westrelin S., P. Mériaux, P. Tabary et Y. Aubert, 2012: Hydrometeorological risks in Mediterranean mountainous areas RHYTMME Project: Risk Management based on a Radar Network. 7th European Conference on Radar in Meteorology and Hydrology (Toulouse, France), 24-29 June 2012. 